\title{
Online Estimation of Ship Dynamic Flexure Model Parameters for Transfer Alignment
}

\author{
Wei Wu, Sheng Chen, Fellow, IEEE, and Shiqiao Qin
}

\begin{abstract}
This paper presents an online approach for estimating the dynamic flexure model parameters in shipboard transfer alignment (TA). Traditionally, the application of Kalman filters (KFs) to the TA process is often restricted because of the lack of real-time information on dynamic flexure characteristics, and a KF designed on the basis of inaccurate parameters of the dynamic flexure model will result in a large alignment error. To overcome this difficulty, a parameter estimation algorithm is proposed in this paper, which utilizes the angular increment difference measured by the master inertial navigation system (MINS) and the slave inertial navigation system. Specifically, the Tufts-Kumaresan method is introduced to compute the unknown parameters of the dynamic flexure model from the angular increment correlation function. Our simulation results show that the proposed method can estimate the dynamic flexure parameters with a high degree of accuracy, even in low signalto-noise ratio conditions. This parameter estimation method does not require a priori knowledge of dynamic flexure characteristics and, therefore, provides the shipboard sensors with an accurate and rapid-response capability for alignment with the MINS.
\end{abstract}

Index Terms-Dynamic flexure, Gauss-Markov process, parameter estimation, transfer alignment, Tufts-Kumaresan method.

\section{INTRODUCTION}

$\mathbf{M}$ ODERN large ships are equipped with arrays of peripheral apparatus, such as radars, launching vehicles, and optoelectronic and other sensors, whose attitudes must be aligned to a high degree of accuracy in accordance with the main attitude system when in service. A solution to the shipboard transfer alignment (TA) problem, which uses gyro measurements of the master inertial navigation system (MINS) and the slave inertial navigation system (SINS), has been known for some time [1]-[3]. The common procedure of TA based on angular velocity or attitude is done by successively computing the difference of two gyro measurements through a real-time Kalman filter (KF) [4].

Manuscript received February 22, 2012; revised June 28, 2012; accepted August 17, 2012. Manuscript received in final form August 20, 2012. Date of publication November 12, 2012; date of current version August 12, 2013. Recommended by Associate Editor A. Alessandri.

$\mathrm{W}$. Wu is with the School of Opto-Electronic Science and Engineering, National University of Defense Technology, Changsha 410073, China, and also with the Electronics and Computer Science Department, University of Southampton, Southampton SO17 1BJ, U.K. (e-mail: ww6g11@ecs.soton.ac.uk).

$\mathrm{S}$. Chen is with the Electronics and Computer Science Department, University of Southampton, Southampton SO17 1BJ, U.K., and also with the Faculty of Engineering, King Abdulaziz University, Jeddah 21589, Saudi Arabia (e-mail:sqc@ecs.soton.ac.uk).

S. Qin is with the School of Science, National University of Defense Technology, Changsha 410073, China (e-mail: sqqin8@nudt.edu.cn).

Color versions of one or more of the figures in this paper are available online at http://ieeexplore.ieee.org.

Digital Object Identifier 10.1109/TCST.2012.2214778
However, as the ship's structure is elastic when subjected to wave- or wind-induced loads or a fast maneuvering operation, large dynamic flexure and bending may occur between the MINS and SINS coordinate frames, and this is one of the most important sources of error in TA [5]-[7]. To reduce the influence of dynamic flexure, many authors have studied ship's dynamic flexure modeling and compensation approaches [8]-[11], among which the second-order GaussMarkov process is the most commonly adopted to depict the dynamic flexure according to its time characteristics. These studies [2], [4] have demonstrated that the simple second-order Gauss-Markov process can give acceptable results in many applications. In [8], the sensitivity of KF to the accuracy of the dynamic flexure model is studied, and the results presented show that the estimation error has a strong correlation with the accuracy of the model parameters. In [12], it is shown that the performance of the KF-based TA is strongly linked to the accuracy of the dynamic flexure model employed.

Therefore, the most challenging task when applying a TA procedure with the KF is how to determine the dynamic flexure parameters accurately. Because of the complexity of the ship's structure and dynamics [13], a pure analytical derivation of the dynamic flexure model parameters or dynamic flexure characteristics, such as frequency, magnitude, and damping ratio, is not practical. Traditionally, two alternative approaches have been considered: 1) analytical plus empirical and 2) purely statistical. In an analytical plus empirical method, the unknown parameters are simplified as functions of the correlation time and variance of the dynamic flexure signal. However, the values of the correlation time and variance are typically obtained from experience. Previous works [4], [9], [10] have shown that the performance of the KF is not highly sensitive to the value of the correlation time used. Obviously, this approach is far from ideal and cannot be adapted to various working conditions of shipboard TA. A purely statistical approach uses previous dynamic flexure measurement data to determine the model parameters, typically by a least squares fitting. This method is widely applied in airborne TA [12], [14], [15], because experiments to collect dynamic flexure measurement data can be completed during the design and production process. However, owing to the complexity of the flexure of a ship's structure and sea-wave loading conditions, flexure measurement experiments are difficult and are rarely reported [16].

Therefore, for shipboard TA application, the required dynamic flexure parameters are often calculated using an analytical plus empirical method. The complexity of different ship structures and the randomness of wave-load excitations make it hard if not impossible to obtain an accurate and 
robust model to meet the various requirements and operating environments. The alignment accuracy will inevitably be poor when the exact ship structure and actual work conditions differ from those used in estimating the dynamic flexure model parameters. It is highly advisable to adapt the dynamic flexure model parameters or to determine these parameters according to the specific working conditions, and this is the motivation for our work on online parameter estimation for the dynamic flexure model. More specifically, it is known that the dynamic flexure information exists in the gyro measurement difference between the MINS and SINS and this relationship can be utilized to calculate the unknown parameters. We propose an online parameter estimation method by utilizing the angular increment outputs of two gyro units in order to estimate the dynamic flexure model parameters more accurately and to adapt them under various work environments. In accordance with common practice, the second-order Gauss-Markov process, which has the same form with exponentially damped sinusoid (EDS) signals, is used to depict the dynamic flexure. The Tufts-Kumaresan (TK) method [17], [18] is an efficient technique to solve for the frequencies and damping factor of EDS signals, particularly when the data length is short and the signal-to-noise ratio (SNR) is small. This motivates us to adopt the TK method for online parameter estimation of the dynamic flexure model. The simulation results obtained demonstrate that the parameters identified using the TK method have high accuracy in comparison with their corresponding true values. The proposed parameter estimation method requires no a priori knowledge of the dynamic flexure characteristics and works well even under low SNR conditions.

The rest of this paper is organized as follows. In Section II, the ship misalignment angle measurement method and the Kalman filtering function are introduced. Section III presents the TK method for determining the parameters of the dynamic flexure model. Following this, simulation experiments are presented in Section IV. Finally, our conclusions and remarks are offered in Section V.

\section{TA ApProach AND Dynamic Flexure Model}

In this section, the angular velocity matching method as a basic technique in the TA procedure is introduced. Following this, the dynamic flexure model and the KF function are presented.

\section{A. Angular Increment Matching Function}

Suppose that the MINS and SINS are each equipped with three orthogonal ring-laser gyros. As illustrated in Fig. 1, the MINSs coordinates $O_{m}\left(x_{m}, y_{m}, z_{m}\right)$ have been aligned with the ship's body frame ( $b$-frame), and the SINSs coordinates $O_{s}\left(x_{s}, y_{s}, z_{s}\right)$ are in accordance with the peripheral apparatus frame ( $s$-frame). The total misalignment Euler angle between the MINS and SINS is $\varphi(t)$, which includes a static component $\boldsymbol{\phi}_{0}$ and a dynamic component $\boldsymbol{\theta}(t)$. If the misalignment angle is small, $\varphi(t)$ is related to $\phi_{0}$ and $\boldsymbol{\theta}(t)$ by

$$
\boldsymbol{\varphi}(t)=\phi_{0}+\boldsymbol{\theta}(t) .
$$

The MINS measures the ship inertial angular velocity projected onto the $O_{m}\left(x_{m}, y_{m}, z_{m}\right)$ coordinates, which can

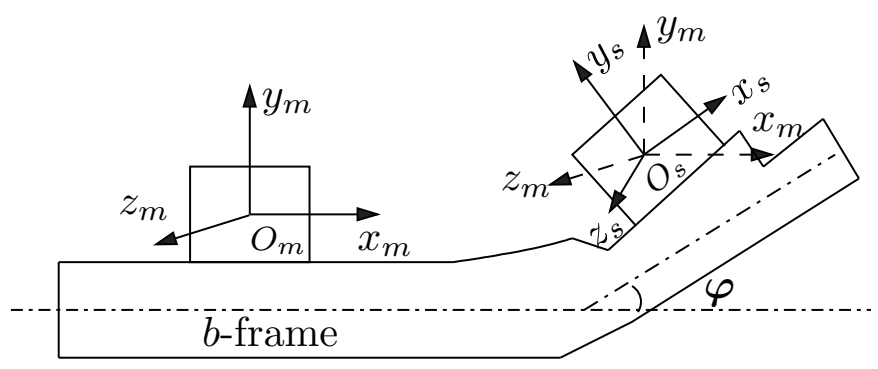

Fig. 1. Schematic diagram of a ship's angular measurement.

be written as $\vec{\Omega}_{i b}^{b}$, while the SINS measures the ship inertial angular velocity projected onto the $O_{s}\left(x_{s}, y_{s}, z_{s}\right)$ coordinates, denoted as $\vec{\Omega}_{i b}^{s}$. The angular velocity relationship can be expressed as [8]

$$
\vec{\Omega}_{i b}^{s}=C_{b}^{s}(\varphi) \vec{\Omega}_{i b}^{b}+\dot{\vec{\theta}}
$$

where for notational simplicity we have dropped time $t$ from $\varphi, C_{b}^{s}(\varphi)$ denotes the transform cosine matrix from the $b$-frame to the $s$-frame, $\dot{\vec{\theta}}$ is an additional velocity caused by the dynamic flexure of the $s$-frame relative to the $b$-frame, and the dot operator $(\dot{\bullet})$ represents differentiation with respect to time $t$.

The difference between the angular velocities measured by the MINS and SINS with respect to the $b$-frame is therefore given by

$$
\Delta \overrightarrow{\boldsymbol{\Omega}}=\overrightarrow{\boldsymbol{\Omega}}_{i b}^{b}-\vec{\Omega}_{i b}^{s}=\left[\boldsymbol{I}_{3}-\boldsymbol{C}_{b}^{s}(\boldsymbol{\varphi})\right] \vec{\Omega}_{i b}^{b}-\dot{\vec{\theta}}
$$

where $\boldsymbol{I}_{3}$ denotes the $3 \times 3$ identity matrix. If the misalignment angle is small, (3) can be approximated as

$$
\Delta \overrightarrow{\mathbf{\Omega}} \approx \widehat{\mathbf{\Omega}}_{i b}^{b} \boldsymbol{\varphi}-\dot{\vec{\theta}}
$$

where $\widehat{\boldsymbol{\Omega}}_{i}^{b}$ is a skew-symmetric matrix of the form

$$
\widehat{\boldsymbol{\Omega}}_{i b}^{b}=\left[\begin{array}{ccc}
0 & \Omega_{i b z}^{b} & -\Omega_{i b y}^{b} \\
-\boldsymbol{\Omega}_{i b z}^{b} & 0 & \boldsymbol{\Omega}_{i b x}^{b} \\
\boldsymbol{\Omega}_{i b y}^{b} & -\boldsymbol{\Omega}_{i b x}^{b} & 0
\end{array}\right]
$$

where $\left(\Omega_{i b x}^{b}, \Omega_{i b y}^{b}, \Omega_{i b z}^{b}\right)$ are the three coordinate values of $\vec{\Omega}_{i b}^{b}$. It can be seen from (4) and (5) that the rank of $\widehat{\boldsymbol{\Omega}}_{i b}^{b}$ is $r=2$, while the dimension of the vectors involved is $n=3$. Since $r<n$, the differential equation (4) has no analytical solution. One approach to solve this problem is by processing through a KF based on an accuracy dynamic flexure model.

\section{B. Ship Dynamic Flexure Model}

A ship's dynamic flexure is caused by its motion due to waves and maneuvers and the vibration due to a variety of sources, and can be depicted using a second-order GaussMarkov process [2], [4], which has a correlation function of the form

$$
R_{\theta_{i}}(\tau)=\sigma_{i}^{2} \exp \left(-\alpha_{i}|\tau|\right)\left(\cos \beta_{i} \tau+\frac{\alpha_{i}}{\beta_{i}} \sin \beta_{i}|\tau|\right)
$$

where $i$ indicates the $x, y$, or $z$ coordinate, $\tau$ is the time lag, $\sigma_{i}^{2}$ is the variance of the $i$-coordinate component of the dynamic flexure, $\alpha_{i}$ is the damping factor, and $\beta_{i}$ is the circular 
frequency. The differential equation representing the ship's dynamic flexure with the correlation function given in (6) can be written as

$$
\ddot{\theta}_{i}+2 \alpha_{i} \dot{\theta}_{i}+b_{i}^{2} \theta_{i}=2 b_{i} \sigma_{i} \sqrt{\alpha_{i}} e_{i}(t)
$$

where $b_{i}^{2}=\alpha_{i}^{2}+\beta_{i}^{2}$ is the square of the prevailing variation frequency and $e_{i}(t)$ is a Gaussian white noise (WN) with unit variance.

Given the parameters of $\alpha_{i}, \beta_{i}$, and $\sigma_{i}$, the optimal KF for TA can be determined. However, the characteristics of the dynamic flexure are often unknown. In most traditional studies, these parameters were estimated from previous ship trials or numerical analysis, which depend on many factors, such as the ship's structure, the sea station, ship's speed, and so on. The main contribution of this paper is to propose an online parameter estimation method for determining these parameters, so that the parameters used in the KF-based TA represent the true dynamic flexure model of the operating conditions that the ship is experiencing.

\section{Kalman Filtering Function}

For TA processing with a KF, the measurement function for (4) is presented in a standard matrix form as

$$
z=H x+v
$$

where $z$ and $v$ are the $3 \times 1$ measurement and measurement error vector, respectively, while $\boldsymbol{H}$ and $\boldsymbol{x}$ denote the measurement matrix and the state vector, respectively. According to (4), the state vector is of size $9 \times 1$, specified by

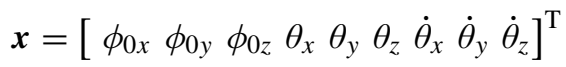

where $\mathrm{T}^{\mathrm{T}}$ denotes the vector or matrix transpose operator, $\left(\phi_{0 x}, \phi_{0 y}, \phi_{0 z}\right),\left(\theta_{x}, \theta_{y}, \theta_{z}\right)$, and $\left(\dot{\theta}_{x}, \dot{\theta}_{y}, \dot{\theta}_{z}\right)$ are the three coordinate values of $\boldsymbol{\phi}_{0}, \boldsymbol{\theta}$, and $\dot{\vec{\theta}}$, respectively, while the $3 \times 9$ measurement matrix is given by

$$
\boldsymbol{H}=\left[\widehat{\boldsymbol{\Omega}}_{i b}^{b} \widehat{\boldsymbol{\Omega}}_{i b}^{b}-\boldsymbol{I}_{3}\right] .
$$

The state equation for the KF is defined as

$$
\dot{\boldsymbol{x}}=\boldsymbol{F} \boldsymbol{x}+\boldsymbol{w}
$$

where the state-space equation matrix $\boldsymbol{F}$ takes the form

$$
\boldsymbol{F}=\left[\begin{array}{ll}
\boldsymbol{O}_{3 \times 3} & \boldsymbol{O}_{3 \times 6} \\
\boldsymbol{O}_{6 \times 3} & \boldsymbol{F}_{6 \times 6}^{1}
\end{array}\right]
$$

with $\boldsymbol{O}_{l \times m}$ denoting the $l \times m$ zero matrix, and

$$
\boldsymbol{F}_{6 \times 6}^{1}=\left[\begin{array}{cccccc}
\boldsymbol{O}_{3 \times 3} & \multicolumn{5}{c}{\boldsymbol{I}_{3}} \\
-b_{x}^{2} & 0 & 0 & -2 \alpha_{x} & 0 & 0 \\
0 & -b_{y}^{2} & 0 & 0 & -2 \alpha_{y} & 0 \\
0 & 0 & -b_{z}^{2} & 0 & 0 & -2 \alpha_{z}
\end{array}\right] .
$$

The $9 \times 1$ state noise vector $\boldsymbol{w}$ has the covariance matrix

$$
E\left[\boldsymbol{w} \boldsymbol{w}^{\mathrm{T}}\right]=\operatorname{diag}\{\underbrace{0, \cdots, 0}_{6}, 4 b_{x}^{2} \sigma_{x}^{2} \alpha_{x}, 4 b_{y}^{2} \sigma_{y}^{2} \alpha_{y}, 4 b_{z}^{2} \sigma_{z}^{2} \alpha_{z}\}
$$

where $E[\bullet]$ denotes the expectation operator and $\operatorname{diag}\{\bullet\}$ is the diagonal matrix.
In the procedure of TA, the KF acts as an observer, and the misalignment angle between the MINS and SINS frames can be optimally estimated by utilizing the dynamic flexure model. Let $\varphi$ be the true misalignment angle between the MINS and SINS, and $\widehat{\varphi}$ its estimate provided by the KF. The TA accuracy is specified by the alignment error vector

$$
\Delta \varphi=\left[\begin{array}{lll}
\Delta \varphi_{x} & \Delta \varphi_{y} & \Delta \varphi_{x}
\end{array}\right]^{\mathrm{T}}
$$

which can be evaluated as follows. Define the alignment error matrix

$$
\boldsymbol{E}_{b}^{s}(\Delta \boldsymbol{\varphi})=\boldsymbol{C}_{b}^{s}(\boldsymbol{\varphi})\left(\boldsymbol{C}_{b}^{s}(\widehat{\boldsymbol{\varphi}})\right)^{\mathrm{T}} .
$$

Then the alignment error vector is given by

$$
\begin{aligned}
\Delta \varphi_{x} & =\arcsin \left(\left.\boldsymbol{E}_{b}^{s}(\Delta \boldsymbol{\varphi})\right|_{3,2}\right) \\
\Delta \varphi_{y} & =\arctan \left(\frac{-\left.\boldsymbol{E}_{b}^{s}(\Delta \boldsymbol{\varphi})\right|_{3,1}}{\left.\boldsymbol{E}_{b}^{s}(\Delta \boldsymbol{\varphi})\right|_{3,3}}\right) \\
\Delta \varphi_{z} & =\arctan \left(\frac{-\left.\boldsymbol{E}_{b}^{s}(\Delta \boldsymbol{\varphi})\right|_{1,2}}{\left.\boldsymbol{E}_{b}^{s}(\Delta \boldsymbol{\varphi})\right|_{2,2}}\right)
\end{aligned}
$$

where $\left.\boldsymbol{E}_{b}^{s}\right|_{i, j}$ denotes the element of $\boldsymbol{E}_{b}^{s}$ at the $i$ th row and $j$ th column. Obviously, the alignment accuracy depends on the accuracy of the dynamic flexure model parameters $\alpha_{i}, \beta_{i}$, and $\sigma_{i}$ used.

\section{Dynamic Flexure Model Parameter Estimation}

In this section, a parameter estimation approach for the dynamic flexure model is proposed in which the TK method estimates the dynamic flexure model parameters based on the MINS and SINS gyro measurement samples.

\section{A. Correlation Function for Parameter Estimation}

As can be seen from (4), the dynamic flexure information is contained in the angular velocity matching function. In order to obtain the dynamic flexure model, an approximate relationship between the gyro outputs and the dynamic flexure angle is required. Since for the ring-laser gyros the outputs are the discrete-time angular increment signals, we first derive the discrete-time increment matching function based on (4). Assuming that the sampling time period $\Delta T$ is sufficiently small and by integrating the both sides of (4) over the time interval $\left(t_{k-1}, t_{k-1}+\Delta T\right)$, we obtain the angular increment matching function as

$$
\Delta \boldsymbol{\Theta}_{k} \approx \widehat{\boldsymbol{\Theta}}_{k} \boldsymbol{\phi}_{0}+\left(\frac{1}{2} \widehat{\boldsymbol{\Theta}}_{k}-\boldsymbol{I}_{3}\right) \boldsymbol{\theta}_{k}+\left(\frac{1}{2} \widehat{\boldsymbol{\Theta}}_{k}+\boldsymbol{I}_{3}\right) \boldsymbol{\theta}_{k-1}
$$

where

$$
\Delta \boldsymbol{\Theta}_{k}=\int_{t_{k-1}}^{t_{k-1}+\Delta T} \Delta \vec{\Omega} d t
$$

is the angular increment difference between the MINS and SINS gyro outputs, and $\widehat{\boldsymbol{\Theta}}_{k}$ is a skew-symmetric matrix with the same form as (5), but whose components are the three coordinates of the MINS gyro increment at $t_{k}$ given by

$$
\boldsymbol{\Theta}_{k}=\int_{t_{k-1}}^{t_{k-1}+\Delta T} \vec{\Omega}_{i b}^{b} d t=\left[\begin{array}{lll}
\Theta_{k x} & \Theta_{k y} & \Theta_{k z}
\end{array}\right]^{\mathrm{T}}
$$


namely,

$$
\widehat{\boldsymbol{\Theta}}_{k}=\left[\begin{array}{ccc}
0 & \Theta_{k z} & -\Theta_{k y} \\
-\Theta_{k z} & 0 & \Theta_{k x} \\
\Theta_{k y} & -\Theta_{k x} & 0
\end{array}\right]
$$

where $\boldsymbol{\theta}_{k}$ and $\boldsymbol{\theta}_{k-1}$ denote the dynamic flexure angles at $t_{k}$ and $t_{k-1}$, respectively.

According to [2] and [16], typical magnitude of a ship's angular motion can be up to 10 degrees, while the amplitude of dynamic flexure can reach several millirads, with a corresponding period of several seconds. In high-accuracy TA, the course alignment in the TA procedure can estimate the static component $\phi_{0}$ to an accuracy of several millirads and compensate it. Taking into account these conditions, the first term on the right-hand side of (20) may be removed, yielding

$$
\Delta \boldsymbol{\Theta}_{k} \approx\left(\frac{1}{2} \widehat{\boldsymbol{\Theta}}_{k}-\boldsymbol{I}_{3}\right) \boldsymbol{\theta}_{k}+\left(\frac{1}{2} \widehat{\boldsymbol{\Theta}}_{k}+\boldsymbol{I}_{3}\right) \boldsymbol{\theta}_{k-1} .
$$

Furthermore, the above-mentioned conditions also ensure that $\left|\Theta_{k x}\right| \ll 1,\left|\Theta_{k y}\right| \ll 1$, and $\left|\Theta_{k z}\right| \ll 1$, and, therefore, (21) can be further approximated as

$$
\Delta \boldsymbol{\Theta}_{k} \approx-\boldsymbol{\theta}_{k}+\boldsymbol{\theta}_{k-1}
$$

It is worth emphasizing that (21) and, hence, (22) are derived from (20) by compensating $\widehat{\boldsymbol{\Theta}}_{k} \boldsymbol{\phi}_{0}$, assuming that the static component $\boldsymbol{\phi}_{0}$ is known.

We now investigate the relationship between the correlation function of $\Delta \boldsymbol{\Theta}_{k}$ given in (22) and that of the second-order Gauss-Markov process. Recall that the latter, which is used to model the dynamic flexure, is given by (6). Considering $\tau \geq 0$, (6) can be transformed as

$R_{\theta_{i}}(\tau)=\frac{\beta_{i} \sigma_{i}^{2}}{\sqrt{\alpha_{i}^{2}+\beta_{i}^{2}}} \exp \left(-\alpha_{i} \tau\right) \sin \left(\beta_{i} \tau+\varepsilon_{i}\right), \quad$ for $\tau \geq 0$

in which $\varepsilon_{i}=\arctan \left(\beta_{i} / \alpha_{i}\right)$. Define

$$
\tilde{R}_{\theta_{i}}(\tau)=\frac{\beta_{i} \sigma_{i}^{2}}{\sqrt{\alpha_{i}^{2}+\beta_{i}^{2}}} \exp \left(-\alpha_{i} \tau+j\left(\beta_{i} \tau+\varepsilon_{i}\right)\right)
$$

where $j=\sqrt{-1}$, and $i$ indicates the $x, y$, or $z$ coordinate, we have

$$
R_{\theta_{i}}(\tau)=\frac{1}{2 j}\left(\tilde{R}_{\theta_{i}}(\tau)-\tilde{R}_{\theta_{i}}^{*}(\tau)\right)
$$

in which ${ }^{*}$ denotes the complex conjugate. If we denote the correlation operator by $\langle\bullet, \bullet\rangle$, then the discrete correlation function of $\Delta \boldsymbol{\Theta}_{k}$ is given by

$$
\begin{aligned}
\boldsymbol{R}_{\Delta \boldsymbol{\Theta}}(n)= & \left\langle\Delta \boldsymbol{\Theta}_{k}, \Delta \boldsymbol{\Theta}_{k+n}\right\rangle \\
= & \left\langle\boldsymbol{\theta}_{k}, \boldsymbol{\theta}_{k+n}\right\rangle+\left\langle\boldsymbol{\theta}_{k-1}, \boldsymbol{\theta}_{k+n-1}\right\rangle \\
& -\left\langle\boldsymbol{\theta}_{k-1}, \boldsymbol{\theta}_{k+n}\right\rangle-\left\langle\boldsymbol{\theta}_{k}, \boldsymbol{\theta}_{k+n-1}\right\rangle \\
= & 2 \boldsymbol{R}_{\boldsymbol{\theta}}(n)-\boldsymbol{R}_{\boldsymbol{\theta}}(n-1)-\boldsymbol{R}_{\boldsymbol{\theta}}(n+1)
\end{aligned}
$$

where $\boldsymbol{R}_{\boldsymbol{\theta}}(n)=\left[\begin{array}{lll}R_{\theta_{x}}(n) & R_{\theta_{y}}(n) \quad R_{\theta_{z}}(n)\end{array}\right]^{\mathrm{T}} \quad$ is the correlation function of $\boldsymbol{\theta}_{k}$, which is given by (25), with $n$ denoting the lag step. With the notation of
$\tilde{\boldsymbol{R}}_{\Delta \Theta}(n)=\left[\begin{array}{lll}\tilde{R}_{\Delta \Theta_{x}}(n) & \tilde{R}_{\Delta \Theta_{y}}(n) & \tilde{R}_{\Delta \Theta_{z}}(n)\end{array}\right]^{\mathrm{T}}$ and using $i$ to indicate the $x, y$, or $z$ coordinate again, we have

$$
\begin{aligned}
\tilde{R}_{\Delta \Theta_{i}}(n) & =2 \tilde{R}_{\theta_{i}}(n)-\tilde{R}_{\theta_{i}}(n-1)-\tilde{R}_{\theta_{i}}(n+1) \\
& =2 c_{i} \exp \left(\left(-\alpha_{i}+j \beta_{i}\right) n\right)
\end{aligned}
$$

with

$$
c_{i}=\frac{\beta_{i} \sigma_{i}^{2}}{\sqrt{\alpha_{i}^{2}+\beta_{i}^{2}}} \exp \left(j \varepsilon_{i}\right)\left(1-\cosh \left(-\alpha_{i}+j \beta_{i}\right)\right) .
$$

Substituting (25) and (27) in (26), we obtain the correlation function for the angular increment difference as

$$
\boldsymbol{R}_{\Delta \Theta}(n)=\frac{1}{2 j}\left(\tilde{\boldsymbol{R}}_{\Delta \Theta}(n)-\tilde{\boldsymbol{R}}_{\Delta \Theta}^{*}(n)\right) .
$$

It can be clearly seen that the correlation function of the angular increment difference $\Delta \boldsymbol{\Theta}_{k}$ has the same form as that of the dynamic flexure angle $\boldsymbol{\theta}_{k}$. Furthermore, $\alpha_{i}$ and $\beta_{i}$ solved from (29) are the damping factors and circular frequencies of the dynamic flexure model, respectively, while the corresponding variances $\sigma_{i}^{2}$ can be evaluated through (28).

\section{B. Tufts-Kumaresan Method}

The TK method is a common choice to resolve closely spaced sinusoids, particularly when the data length is short and the SNR is small, and it is considered to be among the best methods based on the linear prediction approach [19]. The TK algorithm [17] is briefly summarized below.

Given $N$ samples of a sequence $y(n)$ which is assumed to be composed of a sum of $M$ EDS signals

$$
y(n)=\sum_{l=1}^{M} a_{l} \exp \left(s_{l} n\right)+q_{n}, \quad n=1,2, \ldots, N
$$

where $a_{l}$ is the complex amplitude of the damped mode $\exp \left(s_{l}\right)=\exp \left(-\alpha_{l}+j \beta_{l}\right)$ with $\alpha_{l}>0$, and $q_{n}$ is the unknown WN with variance $\sigma_{q}^{2}$. Using the complex conjugate data to set up the backward prediction function, we have

$$
A b=h
$$

where

$$
\begin{aligned}
& \boldsymbol{A}=\left[\begin{array}{cccc}
y^{*}(2) & y^{*}(3) & \cdots & y^{*}(L+1) \\
y^{*}(3) & y^{*}(4) & \cdots & y^{*}(L+2) \\
\vdots & \vdots & \vdots & \vdots \\
y^{*}(N-L+1) & y^{*}(N-L+2) & \cdots & y^{*}(N)
\end{array}\right] \\
& \boldsymbol{b}=\left[\begin{array}{llll}
b_{1} & b_{2} & \cdots & b_{L}
\end{array}\right]^{\mathrm{T}} \\
& \boldsymbol{h}=\left[\begin{array}{llll}
y^{*}(1) & y^{*}(2) & \cdots & y^{*}(N-L)
\end{array}\right]^{\mathrm{T}} \text {. }
\end{aligned}
$$

The prediction error filter polynomial

$$
B(z)=1+b_{1} z^{-1}+b_{2} z^{-2}+\cdots+b_{L} z^{-L}
$$

has zeros at $\exp \left(-s_{l}^{*}\right), l=1,2, \ldots, M$ if $L$ is chosen to satisfy the inequality $M \leqslant L \leqslant N-M$.

Let the singular value decomposition of the data matrix $\boldsymbol{A}$ be given by

$$
\boldsymbol{A}=\boldsymbol{U} \boldsymbol{\Lambda} \boldsymbol{V}^{\mathrm{H}}
$$




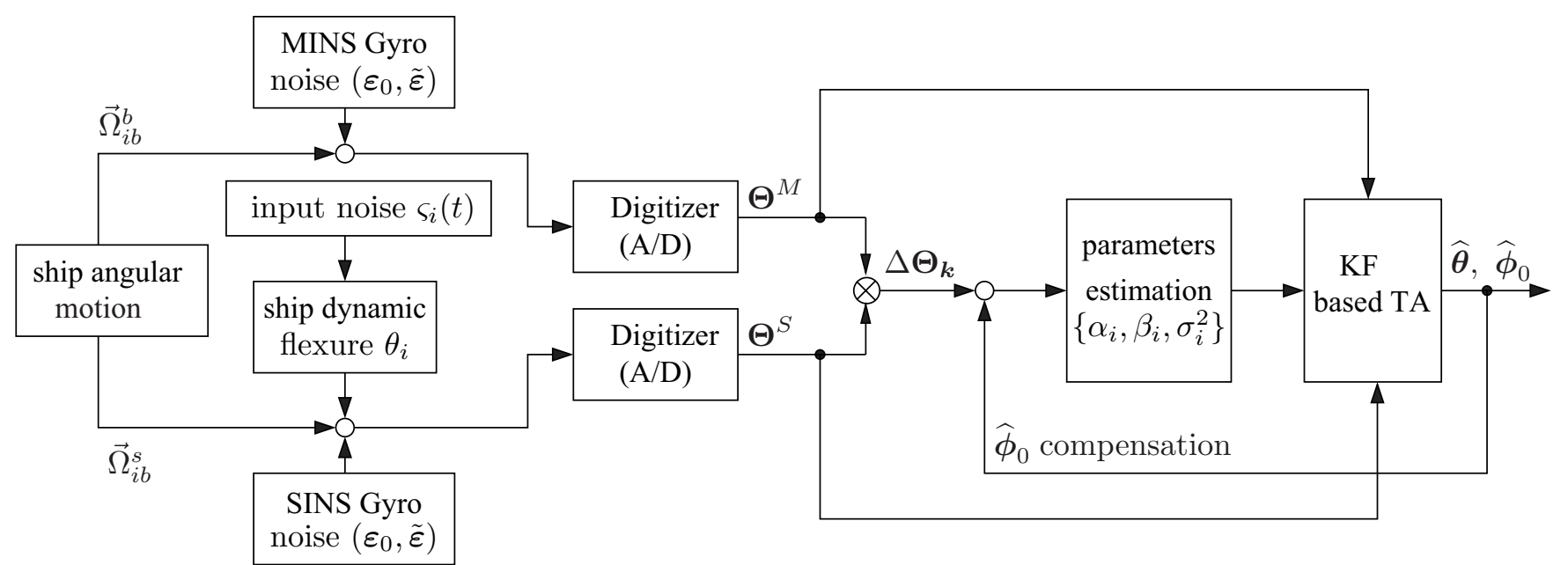

Fig. 2. Schematic diagram of a gyro signal sample generation and dynamic flexure parameter estimation.

where ${ }^{\mathrm{H}}$ stands for the matrix complex conjugate transpose, and the diagonal elements $\lambda_{l}, 1 \leq l \leq L$, of the $(N-L) \times L$ diagonal matrix $\boldsymbol{\Lambda}$ are the singular values of $\boldsymbol{A}$, while the columns of the $(N-L) \times(N-L)$ unitary matrix $\boldsymbol{U}$ are the eigenvectors of $\boldsymbol{A} \boldsymbol{A}^{\mathrm{H}}$, and the columns of the $L \times L$ unitary matrix $\boldsymbol{V}$ are the eigenvectors of $\boldsymbol{A}^{\mathrm{H}} \boldsymbol{A}$. Using the first $M$ dominant nonzero singular values of $\boldsymbol{A},\left\{\lambda_{l}\right\}_{l=1}^{M}$, the coefficient vector of the prediction error filter is calculated by

$$
\boldsymbol{b}=-\sum_{l=1}^{M} \lambda_{l}^{-1} \boldsymbol{u}_{l}^{\mathrm{H}} \boldsymbol{h} \boldsymbol{v}_{l}
$$

where $\boldsymbol{u}_{l}$ and $\boldsymbol{v}_{l}$ denote the associated eigenvectors of $\boldsymbol{A} \boldsymbol{A}^{\mathrm{H}}$ and $\boldsymbol{A}^{\mathrm{H}} \boldsymbol{A}$, respectively. Finding the roots of the prediction error filter polynomial of (35) yields the set of $M$ zeros from which the $M$ damped modes $\exp \left(s_{l}\right), 1 \leq l \leq M$, can be determined. Then the amplitudes $a_{l}$ can easily be estimated based on the dataset $\{y(n)\}$ described by (30) according to the least squares principle.

\section{Dynamic Flexure Parameter Estimation Procedure}

Comparing (27) with (30), it can be seen that these two functions have the same form. Therefore, the dynamic flexure model parameters can be estimated from the correlation function of (29) using the TK method described previously. Noting that only an estimate $\widehat{\boldsymbol{\phi}}_{0}$ is available for compensating the static misalignment angle component $\boldsymbol{\phi}_{0}$, we have the following the iterative parameter estimation procedure based on the TK method.

1) Initialization: Use the result of the initial course alignment for $\widehat{\boldsymbol{\phi}}_{0}$. Give $I_{\mathrm{T}-\mathrm{K}}$, the number of iterations for the TK method, and set the iteration index $l=0$.

2) TK Method: Set $l=l+1$, and perform the following steps.

a) Calculate the angular increment difference $\Delta \boldsymbol{\Theta}_{k}$ of the MINS and SINS gyro outputs, and compensate the static misalignment angle $\boldsymbol{\phi}_{0}$ using $\widehat{\boldsymbol{\phi}}_{0}$.

b) Calculate the correlation function of the compensated $\Delta \boldsymbol{\Theta}_{k}$, and use (31)-(34) to construct the TK prediction function. c) Find the roots of the prediction error filter polynomial using the TK method and, thereby, obtain the damping factors $\alpha_{i}$ and the angular frequencies $\beta_{i}$.

d) Apply the obtained $\alpha_{i}$ and $\beta_{i}$ in (27) to (29) to calculate the dynamic flexure variances $\sigma_{i}^{2}$.

3) End of TK Method: If $\left(l<I_{\mathrm{T}-\mathrm{K}}\right)$, go to KF based TA; or else, end of the iterative procedure.

4) KF Based TA: With the dynamic flexure parameter estimates $\left\{\alpha_{i}, \beta_{i}, \sigma_{i}^{2}\right\}$, perform the KF-based TA processing, which yields a new estimate $\widehat{\boldsymbol{\phi}}_{0}$. Then go to the TK method.

According to (29), the roots of the prediction error filter polynomial of (35) are in pairs of $\exp \left(-s_{l}^{*}\right)$ and $\exp \left(-s_{l}\right)$, $1 \leq l \leq M$, and we simply use one root from each pair to calculate the dynamic flexure parameters. In the simulation study, we will show that typically $I_{\mathrm{T}-\mathrm{K}}=2$ is sufficient. In other words, two passes of the TK algorithm are sufficient to obtain an accurate estimate of the dynamic flexure parameters.

\section{Simulation Results AND Analysis}

We set up a simulation experiment to investigate the proposed online method for estimating the dynamic flexure parameters.

\section{A. Simulation Experimental Setup}

There exist many error sources that may affect the accuracy of parameters estimation, such as gyro measurement noise and input noise in dynamic flexure signals. These error sources are explicitly taken into account in setting up the simulation system. The diagram of the gyro signal sample generation and parameter estimation scheme is shown in Fig. 2.

The ship's angular motion is obtained by solving the equation of motion [20]

$$
\boldsymbol{M} \ddot{\vec{\xi}}(t)+\boldsymbol{B} \dot{\vec{\xi}}(t)+\boldsymbol{C} \vec{\xi}(t)=\vec{Z}(t)
$$

where $\boldsymbol{M}, \boldsymbol{B}$, and $\boldsymbol{C}$ denote the total mass, damping, and stiffness matrices, respectively, while $\vec{Z}(t)$ denotes the wave excitation force vector and $\vec{\xi}(t)$ denotes the principal coordinate vector of the ship's attitude. We assume that the wave 
TABLE I

Ship's Attitude Parameters Used in the Simulation System

\begin{tabular}{lccc}
\hline & $\begin{array}{c}\text { Magnitude } \\
\sigma_{\xi_{i}}(\mathrm{deg})\end{array}$ & $\begin{array}{c}\text { Frequency } \\
\beta_{\xi_{j}} / 2 \pi(\mathrm{Hz})\end{array}$ & $\begin{array}{c}\text { Damping factor } \\
\alpha_{\xi_{j}}\left(\mathrm{~s}^{-1}\right)\end{array}$ \\
\hline Pitch & 2.20 & 0.18 & 0.10 \\
Roll & 3.40 & 0.07 & 0.06 \\
Yaw & 0.80 & 0.05 & 0.12 \\
\hline
\end{tabular}

TABLE II

True Dynamic Flexure Parameters Used in the SiMULATION SYSTEM

\begin{tabular}{lccc}
\hline & $\begin{array}{c}\text { Magnitude } \\
\sigma_{i}(\mathrm{mrad})\end{array}$ & $\begin{array}{c}\text { Frequency } \\
\beta_{i} / 2 \pi(\mathrm{Hz})\end{array}$ & $\begin{array}{c}\text { Damping factor } \\
\alpha_{i}\left(\mathrm{~s}^{-1}\right)\end{array}$ \\
\hline Pitch & 0.40 & 0.19 & 0.13 \\
Roll & 0.68 & 0.17 & 0.11 \\
Yaw & 0.50 & 0.18 & 0.10 \\
\hline
\end{tabular}

excitation force $\vec{Z}(t)$ is a stationary process and that the hydrodynamic coefficients $\boldsymbol{M}, \boldsymbol{B}$, and $\boldsymbol{C}$ are time-invariant during the simulation process. The correlation function of the ship's angular motion can also been derived from a secondorder Gauss-Markov process, which is given by

$$
R_{\xi_{i}}(\tau)=\sigma_{\xi_{i}}^{2} \exp \left(-\alpha_{\xi_{i}}|\tau|\right)\left(\cos \beta_{\xi_{i}} \tau+\frac{\alpha_{\xi_{i}}}{\beta_{\xi_{i}}} \sin \beta_{\xi_{i}}|\tau|\right)
$$

where $i$ again denotes the $x, y$, or $z$ coordinate, while $\sigma_{\xi_{i}}^{2}$, $\alpha_{\xi_{i}}$, and $\beta_{\xi_{i}}$ denote the variance, damping factor, and circular frequency, respectively, of the $i$ coordinate of the ship's attitude angle. Table I lists the simulation parameters of ship's attitude angles used. The values of $\beta_{\xi_{i}}$ and $\alpha_{\xi_{i}}$ are taken from our real data identification results, while the values of $\sigma_{\xi_{i}}$ are set according to our experimental experience.

The WN process and two-colored noise processes having Pierson-Moskowitz (PM) spectrum [21] and Bretschneider (BS) spectrum [22], respectively, were taken as the wave excitation force $\vec{Z}(t)$ in (38) to simulate the various motion conditions of the ship. Empirical ocean wave spectra, such as the PM spectrum and the BS spectrum, are widely applied in ship engineering. The PM spectrum [21] defined by a spectral peak frequency is given by

$$
S_{P M}(\omega)=\frac{\alpha_{s} g^{2}}{\omega^{5}} \exp \left(-\beta_{s}\left(\frac{\omega_{p}}{\omega}\right)^{4}\right)
$$

where $\omega$ denotes the wave frequency, $\omega_{p}$ is the peak wave frequency, $g$ is the gravity, $\alpha_{s}=8.1 \times 10^{-3}$ is the intensity of the spectrum, and $\beta_{s}=1.25$ is the shape factor. The BS spectrum [22], on the other hand, takes the form

$$
S_{B S}(\omega)=\frac{5 \omega_{p}^{4}}{16 \omega^{5}} H_{1 / 3}^{2} \exp \left(-\frac{5}{4}\left(\frac{\omega_{p}}{\omega}\right)^{4}\right)
$$

where $H_{1 / 3}$ is the significant wave height. We set $\omega_{p}=1 \mathrm{rad} / \mathrm{s}$ in both (40) and (41), and $H_{1 / 3}=0.5 \mathrm{~m}$ in (41) to simulate the ship's angular motion.

The dynamic flexure angle is simulated by (6), and the variances $\sigma_{i}^{2}$, damping factors $\alpha_{i}$, and frequencies $\beta_{i}$ used to simulate the dynamic flexure are listed in Table II. The damping factors and frequencies are identified from our real
TABLE III

PARAmeters of the Gyro Noises USED In the Simulation System. WN STD DENOTES THE STANDARD DEVIATION OF THE WN COMPONENT

\begin{tabular}{ccc|cc}
\hline & \multicolumn{2}{c}{ MINS } & \multicolumn{2}{c}{ SINS } \\
\hline & $\begin{array}{c}\text { Bias } \boldsymbol{\varepsilon}_{0} \\
(\operatorname{deg} / \mathrm{h})\end{array}$ & $\begin{array}{c}\text { Random walk } \\
\tilde{\boldsymbol{\varepsilon}} \text { WN STD } \\
(\mathrm{deg} / \sqrt{\mathrm{h}})\end{array}$ & $\begin{array}{c}\text { Bias } \boldsymbol{\varepsilon}_{0} \\
(\operatorname{deg} / \mathrm{h})\end{array}$ & $\begin{array}{c}\text { Random walk } \\
\tilde{\boldsymbol{\varepsilon}} \text { WN STD } \\
(\mathrm{deg} / \sqrt{\mathrm{h}})\end{array}$ \\
\hline $\mathrm{X}$ & 0.005 & 0.001 & 0.02 & 0.005 \\
$\mathrm{Y}$ & 0.005 & 0.001 & 0.02 & 0.005 \\
$\mathrm{Z}$ & 0.005 & 0.001 & 0.02 & 0.005 \\
\hline
\end{tabular}

measurement data in sea trials, while the values of the variances are set according to our empirical experience to reflect a certain level of real sea condition. Note that the values of these dynamic flexure parameters are very similar to those of the experimental results reported in [16]. Since in reality the dynamic flexure signal is polluted by the noise due to the ship's engine vibration, we add a Gaussian WN $\varsigma_{i}(t)$ of variance $\sigma_{\varsigma_{i}}^{2}$ to the dynamic flexure signal $\theta_{i}$. The SNR of the dynamic flexure signal is then defined by

$$
\mathrm{SNR}_{i}=10 \log _{10} \frac{\sigma_{i}^{2}}{\sigma_{\varsigma_{i}}^{2}}
$$

where $i$ indicates the $x, y$, or $z$ coordinate. The static misalignment angle after the initial course alignment compensation is set as

$$
\boldsymbol{\phi}_{0}=[3.5 \mathrm{mrad} 3.5 \mathrm{mrad} 3.5 \mathrm{mrad}]^{\mathrm{T}} .
$$

The ring-laser gyro signals are subjected to the bias error vector $\boldsymbol{\varepsilon}_{0}$ and the random walk noise vector $\tilde{\boldsymbol{\varepsilon}}$, whose parameters are listed in Table III. The simulated MINS and SINS gyro outputs are then used to estimate the dynamic flexure parameters and are processed through a KF to compute the misalignment angle for TA.

\section{B. Simulation Experimental Results}

Before we present our simulation results, it is worth recapitulating the purpose of identifying the dynamic flexure model parameters. The KF requires the dynamic flexure model to provide an estimate $\widehat{\varphi}$ for the misalignment angle $\varphi$ between the MINS and SINS. The TA accuracy is specified by the alignment error vector $\Delta \varphi$, which can be calculated according to (15)-(19). Many practical applications require a high alignment accuracy. For example, shipboard tactical weapon systems often require the alignment error to be within $0.6 \mathrm{mrad}$. A simulation experiment was conducted to validate the effectiveness of the proposed method using the angular increment difference to estimate the unknown dynamic flexure parameters. We assumed that the total data length for TA was $600 \mathrm{~s}$ with the sample frequency of $20 \mathrm{~Hz}$. All the results presented were obtained by averaging over 100 independent trials in the presence of randomly generated noise for simulating ship attitude, dynamic flexure, and gyro noise signals. The ship's motion data were generated under three conditions, namely, the wave excitation force vector was: 1) a WN process; 2) a colored noise process with a PM spectrum; and 3) a colored noise process with a BS spectrum. 
1) Tuning the TK Method's Parameters Under WN Wave Excitation Force: According to [17], the performance of the TK method depends on its algorithmic parameters $M, N$, and $L$. The gyro sample length $T$ of the sequence $\left\{\Delta \boldsymbol{\Theta}_{k}\right\}$ used to calculate the correlation function $\boldsymbol{R}_{\Delta \boldsymbol{\Theta}}(\tau), 0 \leq \tau \leq N$, for the TK prediction function also obviously influences the performance. Therefore, we first determine the appropriate values for these parameters under the $\mathrm{WN}$ wave excitation force condition. The number of iterations in the iterative TK estimation procedure is set to $I_{\mathrm{TK}}=2$, as we will show that this choice is sufficient.

As indicated in Section III-C, the solution of (35) yields a pair of roots for every damping mode and, therefore, we set $M=2$ in the TK algorithm. After experimenting with different $L$ values in the simulation, an appropriate value for $L$ was found to be $L=6 \mathrm{~s}$ or 120 samples, which corresponded to approximately one period of the dynamic flexure. We also empirically determined the appropriate values for $N$ and $T$ using experiments in which the noise $\varsigma_{i}(t)$ was added to the dynamic flexure signal. Note that samples of the correlation function $\boldsymbol{R}_{\Delta \Theta}(\tau)$ are used to identify the dynamic flexure model parameters. To help selecting an appropriate value of the TK prediction sample length $N$, in Fig. 3 we plot in a typical realization of the correlation function $\boldsymbol{R}_{\Delta \Theta}(\tau)$ given $\mathrm{SNR}_{i}=20 \mathrm{~dB}$, in comparison with the corresponding correlation function realization under the noise-free condition. It can be seen from Fig. 3 that the choice of $N=20 \mathrm{~s}$ or 400 samples may be appropriate, since $\boldsymbol{R}_{\Delta \Theta}(\tau)$ for $\tau>20 \mathrm{~s}$ would mainly contain the noise, particularly under small SNR conditions. Indeed, our extensive experiments confirmed that the TK identification results and the KF-based TA accuracy obtained with $N=20 \mathrm{~s}$ are superior to those obtained with $N=30$ s. Therefore we set the TK prediction sample length $N$ to $20 \mathrm{~s}$ in the simulation. Fig. 4 depicts the mean parameter estimation errors for $\alpha_{i}, \beta_{i}$, and $\sigma_{i}$, as well as the corresponding average alignment error obtained with different lengths of $T$, with $\mathrm{SNR}_{i}=20 \mathrm{~dB}, N=20 \mathrm{~s}, L=6 \mathrm{~s}$, and $M=2$, where vertical lines indicate the related standard deviations. As can be seen from Fig. 4(a)-(c), the estimation accuracy improves (smaller error bars) as the length $T$ increases. Therefore, we set the gyro sample length $T=600 \mathrm{~s}$ for correlation function calculation.

We now show that it is sufficient to set the number of iterations in the iterative TK estimation procedure to $I_{\mathrm{T}-\mathrm{K}}=2$. With $T=600 \mathrm{~s}, N=20 \mathrm{~s}, L=6 \mathrm{~s}, M=2$, and $\mathrm{SNR}=20 \mathrm{~dB}$, we vary $I_{\mathrm{TK}}$ and plot the resulting mean parameter estimation errors for the parameters $\alpha_{i}, \beta_{i}$, and $\sigma_{i}$, as well as the average alignment error, in Fig. 5. It can be clearly seen from Fig. 5(a)-(c) that two iterations are sufficient to achieve a highly accurate estimate, and increasing the number of iterations further does not improve the parameter estimation accuracy. The algorithmic parameters for the iterative TK estimation procedure determined by this extensive tuning under the WN wave excitation force are summarized in Table IV.

2) Tuning the TK Method's Parameters Under Colored Noise Wave Excitation Force: We also performed extensive tuning to determine appropriate algorithmic parameters for

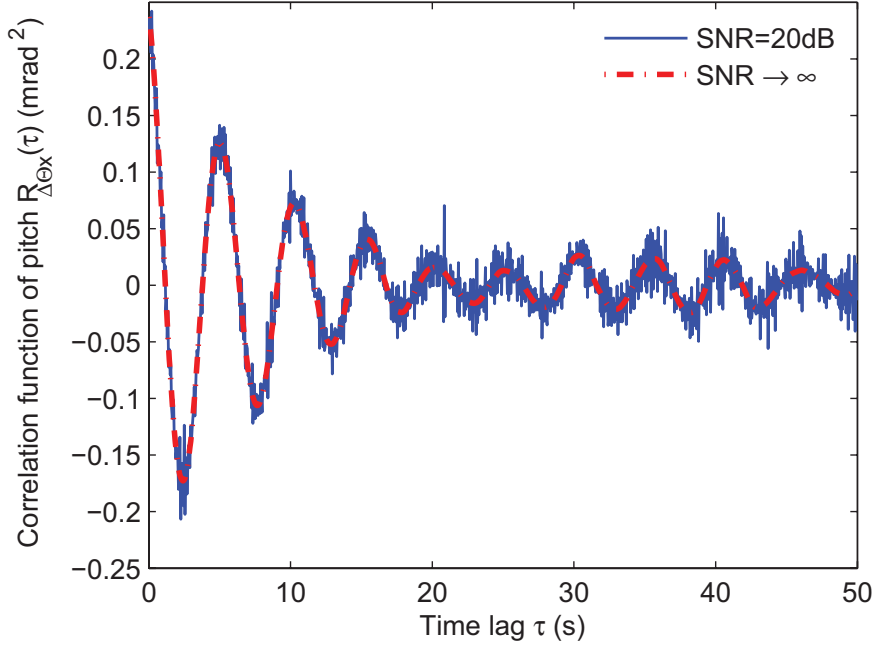

(a)

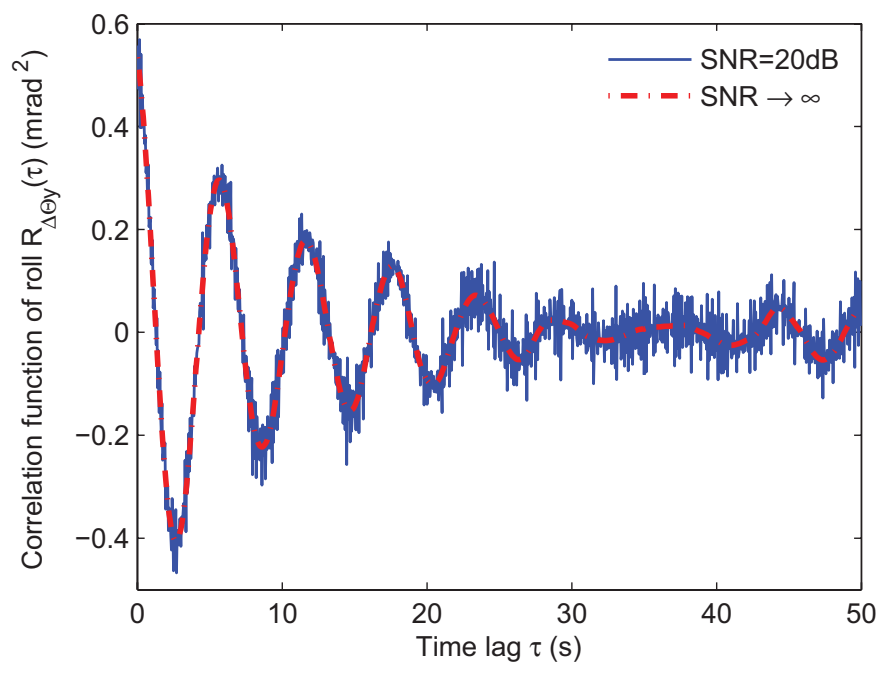

(b)

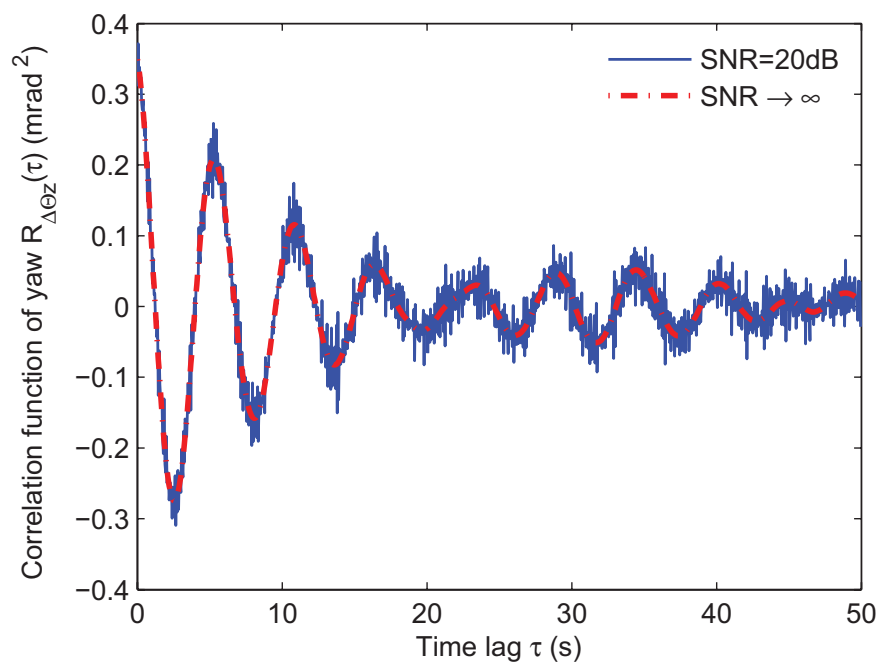

(c)

Fig. 3. Typical realizations of the correlation function $\boldsymbol{R}_{\Delta \Theta}(\tau)$ for SNR $\rightarrow$ $\infty$ and SNR $=20 \mathrm{~dB}$, respectively, given $T=600 \mathrm{~s}$. (a) Pitch. (b) Roll. (c) Yaw. The wave excitation force is a WN.

the iterative TK estimation procedure under the two conditions of colored noise wave excitation forces with the PM and BS spectra, respectively. Our tuning results suggest that 


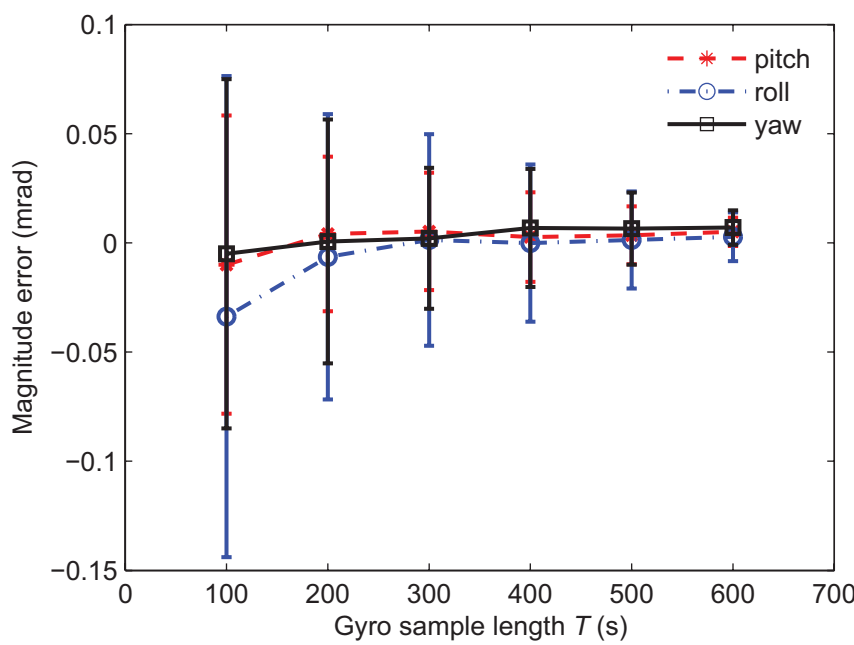

(a)

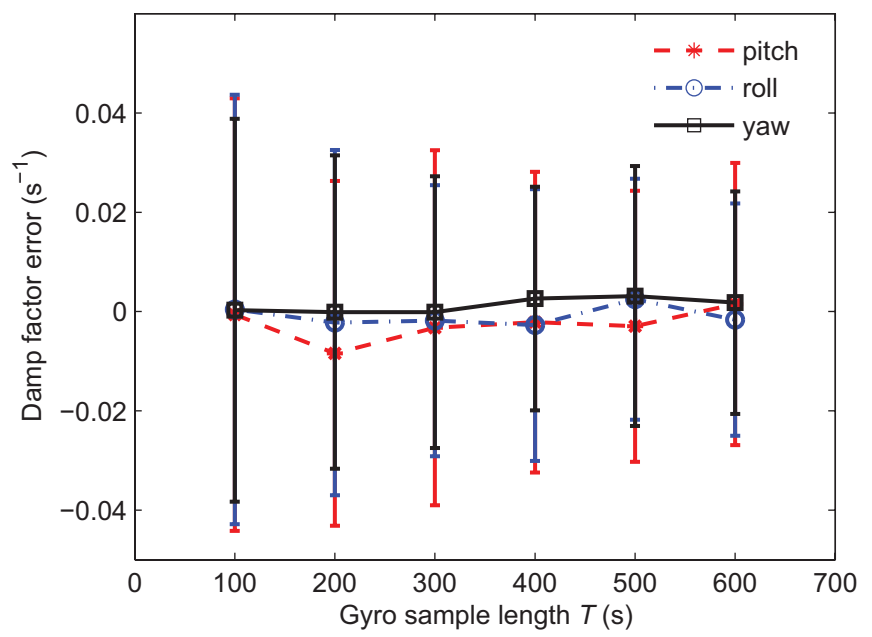

(c)

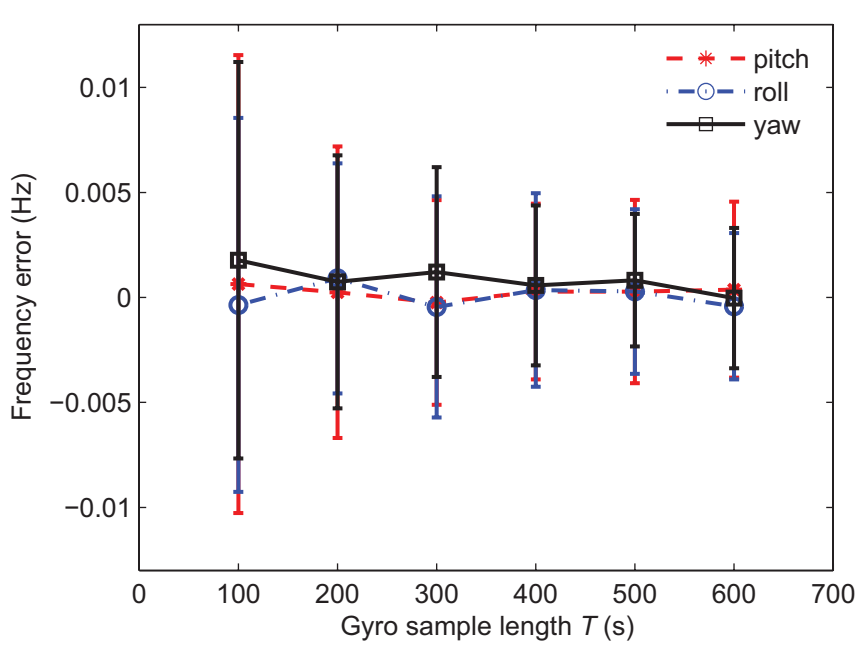

(b)

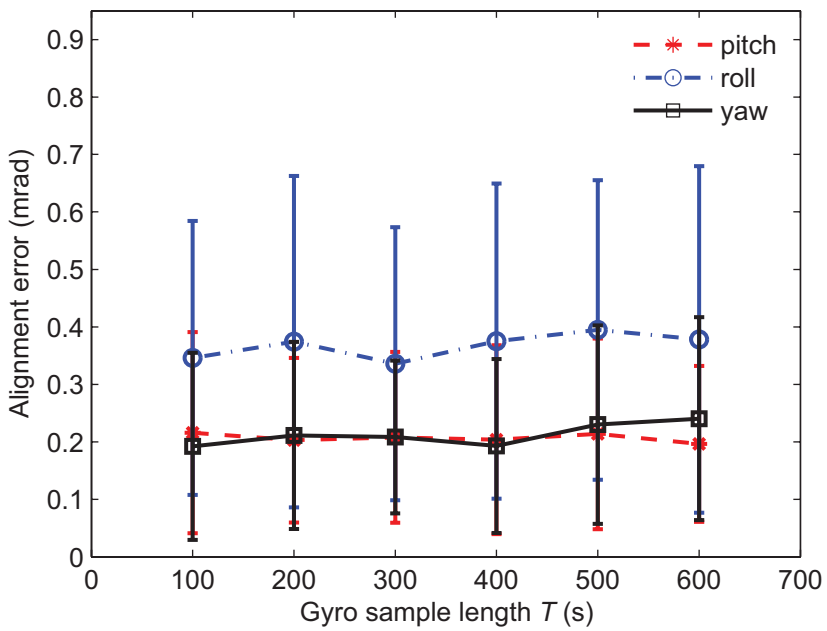

(d)

Fig. 4. Mean parameter estimation errors and average alignment error obtained for different gyro sample lengths $T$, given $\mathrm{SNR}=20 \mathrm{~dB}, I_{\mathrm{T}}-\mathrm{K}=2$, $N=20 \mathrm{~s}, L=6 \mathrm{~s}$, and $M=2$. (a) Error for magnitude $\sigma_{i}$. (b) Error for frequency $\beta_{i} / 2 \pi$. (c) Error for the damping factor $\alpha_{i}$. (d) Average alignment error. The vertical lines indicate the corresponding standard deviations or error bars. The wave excitation force is a WN.

TABLE IV

Algorithmic PARAMETERS OF THE ITERATIVE TK ESTIMATION PROCEDURE USED IN THE SIMULATION EXPERIMENT

\begin{tabular}{l|c|c|c|c}
\hline \hline $\begin{array}{c}\text { TK } \\
\text { Iterations }\end{array}$ & $\begin{array}{c}\text { Gyro sample } \\
\text { length }\end{array}$ & $\begin{array}{c}\text { Data sample } \\
\text { length }\end{array}$ & $\begin{array}{c}\text { Predictor } \\
\text { length }\end{array}$ & $\begin{array}{c}\text { Sinusoid } \\
\text { signals }\end{array}$ \\
\hline \hline \multicolumn{5}{|c}{ WN wave excitation force } \\
\hline$I_{\mathrm{TK}}=2$ & $T=600 \mathrm{~s}$ & $N=20 \mathrm{~s}$ & $L=6 \mathrm{~s}$ & $M=2$ \\
\hline \hline Colored noise wave excitation force with PM spectrum \\
\hline$I_{\mathrm{TK}}=2$ & $T=600 \mathrm{~s}$ & $N=20 \mathrm{~s}$ & $L=8 \mathrm{~s}$ & $M=2$ \\
\hline \hline Colored noise wave excitation force with BS spectrum & \\
\hline$I_{\mathrm{TK}}=2$ & $T=600 \mathrm{~s}$ & $N=20 \mathrm{~s}$ & $L=6 \mathrm{~s}$ & $M=2$ \\
\hline \hline
\end{tabular}

appropriate algorithmic parameters are similar to those determined under the WN wave excitation force condition. Specifically, for the wave excitation force with the PM spectrum, with the exception of the predictor length which should now be chosen as $L=8 \mathrm{~s}$ or 160 samples, the rest of the algorithmic parameters are the same as those for the WN wave excitation force. For the wave excitation force with the BS spectrum, we found that the appropriate algorithmic parameters are identical to those used under the $\mathrm{WN}$ wave excitation force. The algorithmic parameters used in the simulation for the iterative TK estimation procedure under the two colored noise wave excitation forces are also given in Table IV.

3) Estimation Results: We first considered the case of noisefree measurements, namely, $\sigma_{\varsigma_{i}}^{2}=0$ or $\mathrm{SNR}_{i} \rightarrow \infty$. Table V summarizes the means and standard deviations (in bracket) of the estimation results for the parameters $\alpha_{i}, \beta_{i}$, and $\sigma_{i}$ under three different wave excitation forces. Clearly, the parameter estimates obtained by the iterative TK estimation procedure under three different ship motion conditions with the WN and two colored noise wave excitation forces, respectively, are very similar. Compared to the true dynamic flexure parameters given in Table II, it can be seen that the parameter estimates obtained by the TK algorithm are very accurate. The KF-based TA results obtained on the basis of these identified dynamic flexure models under the noise-free measurement condition of $\sigma_{s_{i}}^{2}=0$ are shown in Table VI, where it can be observed that a highly accurate alignment is achieved. In particular, 


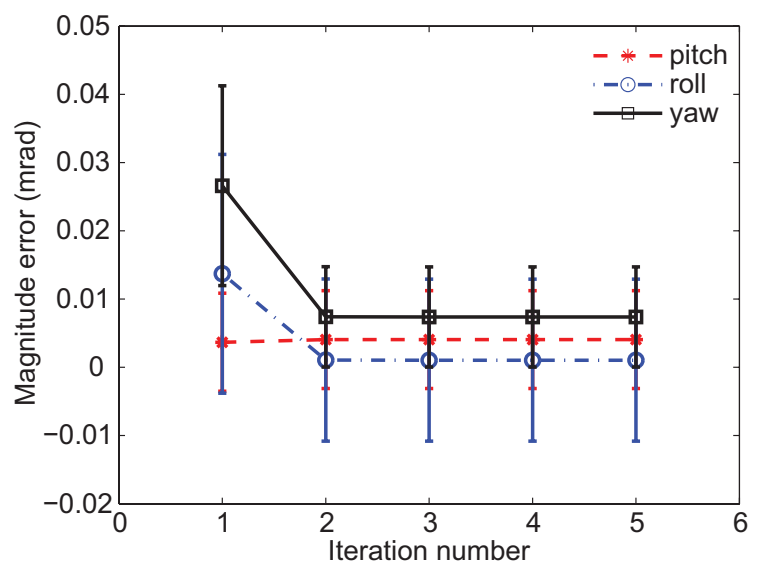

(a)

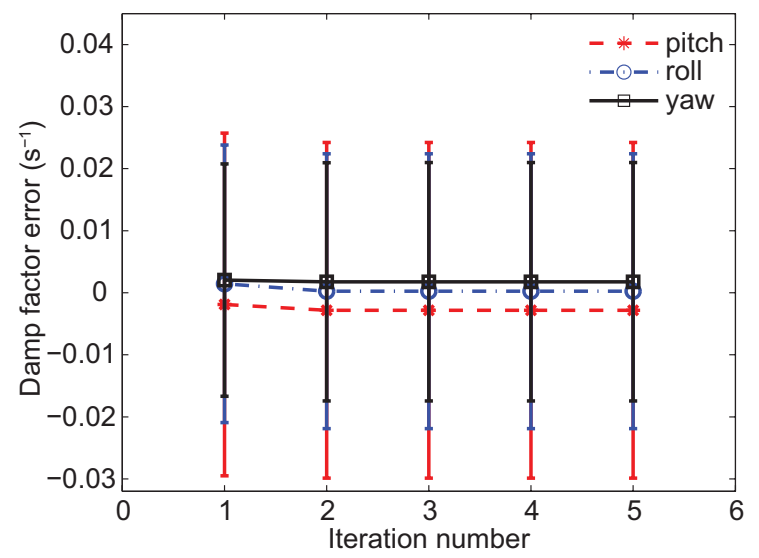

(c)

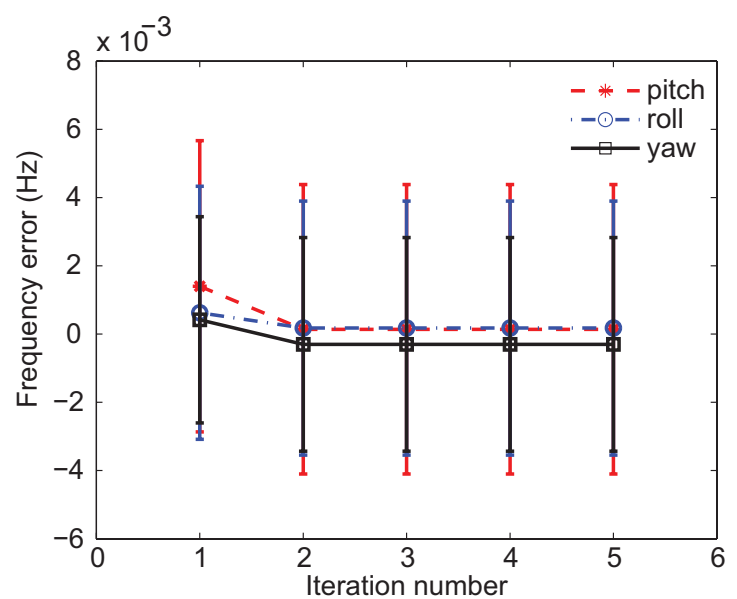

(b)

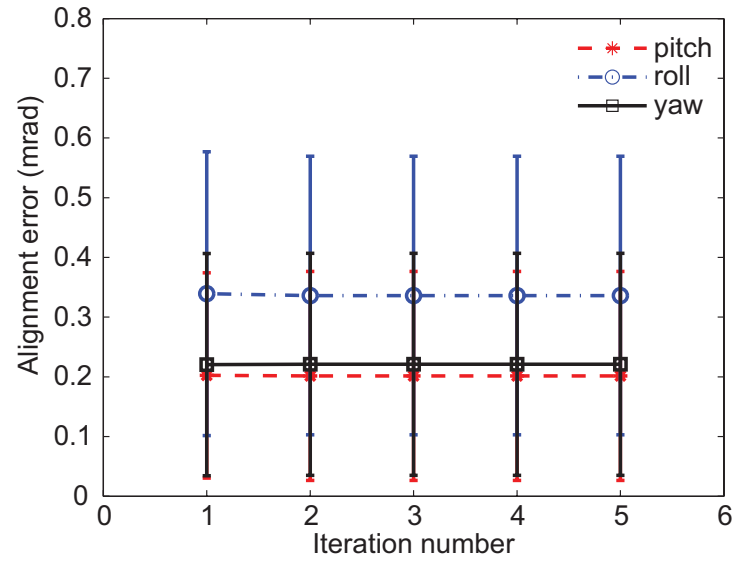

(d)

Fig. 5. Mean parameter estimation errors and average alignment error as functions of the iteration number in the iterative TK estimation procedure, with $T=600 \mathrm{~s}, N=20 \mathrm{~s}, L=6 \mathrm{~s}, M=2$, and SNR $=20 \mathrm{~dB}$. (a) Error for magnitude $\sigma_{i}$. (b) Error for frequency $\beta_{i} / 2 \pi$. (c) Error for damping factor $\alpha_{i}$. (d) Average alignment error. The vertical lines indicate the corresponding standard deviations or error bars. The wave excitation force is a WN.

TABLE V

MEANS AND STANDARd DEVIATIONS OF THE ESTIMATED DyNAMiC FLEXURE PARAMETERS OBTAINED UNDER THE NOISE-FrEE MEASUREMENT CONDITION OF $\sigma_{\varsigma_{i}}^{2}=0$ OR $\mathrm{SNR}_{i} \rightarrow \infty$

\begin{tabular}{l|c|c|c}
\hline \hline & $\begin{array}{c}\text { Magnitude } \\
\sigma_{i}(\mathrm{mrad})\end{array}$ & $\begin{array}{c}\text { Frequency } \\
\beta_{i} / 2 \pi(\mathrm{Hz})\end{array}$ & $\begin{array}{c}\text { Damping factor } \\
\alpha_{i}\left(\mathrm{~s}^{-1}\right)\end{array}$ \\
\hline \hline \multicolumn{5}{c}{ WN wave excitation force } \\
\hline Pitch & $0.4044(0.0067)$ & $0.1898(0.0035)$ & $0.1270(0.0257)$ \\
Roll & $0.6824(0.0110)$ & $0.1699(0.0035)$ & $0.1106(0.0227)$ \\
Yaw & $0.5073(0.0082)$ & $0.1798(0.0032)$ & $0.0992(0.0195)$ \\
\hline \hline Colored noise wave excitation force with PM spectrum \\
\hline Pitch & $0.4055(0.0080)$ & $0.1899(0.0047)$ & $0.1302(0.0271)$ \\
Roll & $0.6806(0.0140)$ & $0.1702(0.0037)$ & $0.1088(0.0205)$ \\
Yaw & $0.5053(0.0088)$ & $0.1799(0.0039)$ & $0.1016(0.0192)$ \\
\hline \hline Colored noise wave excitation force with BS spectrum \\
\hline Pitch & $0.4051(0.0067)$ & $0.1902(0.0043)$ & $0.1314(0.0235)$ \\
Roll & $0.6779(0.0129)$ & $0.1697(0.0038)$ & $0.1141(0.0233)$ \\
Yaw & $0.5057(0.0082)$ & $0.1806(0.0034)$ & $0.1014(0.0214)$ \\
\hline \hline
\end{tabular}

the alignment errors obtained under the ship's motion with the WN wave excitation force were smaller than $0.3 \mathrm{mrad}$. However, the alignment errors obtained under the two colored noise wave excitation forces were larger than those obtained under the WN excitation force. The largest alignment error was in the roll coordinate under the PM spectrum wave excitation force, which had the average value of 0.675 with the standard deviation of 0.4671 .

We next investigated the influence of the noise in the measurement by varying the SNR of the dynamic flexure signal from 12 to $32 \mathrm{~dB}$. For each SNR, we generated the gyro samples under three different motion conditions of the ship to estimate the dynamic flexure parameters, and performed the KF-based TA using the estimated dynamic flexure models. Figs. 6-8 show the average parameter estimation errors for $\sigma_{i}, \alpha_{i}$, and $\beta_{i}$, respectively. It can be seen that the average parameter estimation errors are small, indicating the accuracy of the TK algorithm. More importantly, the average parameter estimation errors and their error bars are similar across the range of the SNR values tested and there is no notable difference in the estimation errors under different ship motion conditions. This demonstrates the robustness of the algorithm in the noise-polluted shipboard environment as well as various ship motion conditions. With the estimated dynamic flexure models, the KF was capable of achieving highly accurate TA in noise-polluted environments, as can be seen in Fig. 9. 


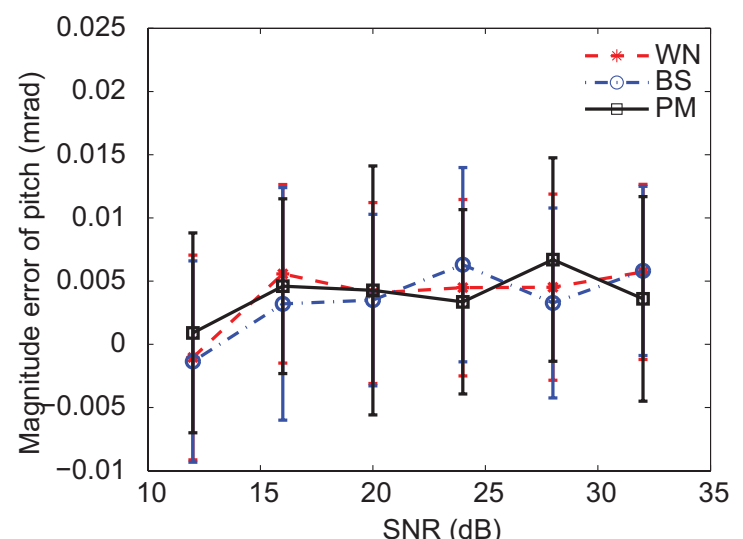

(a)

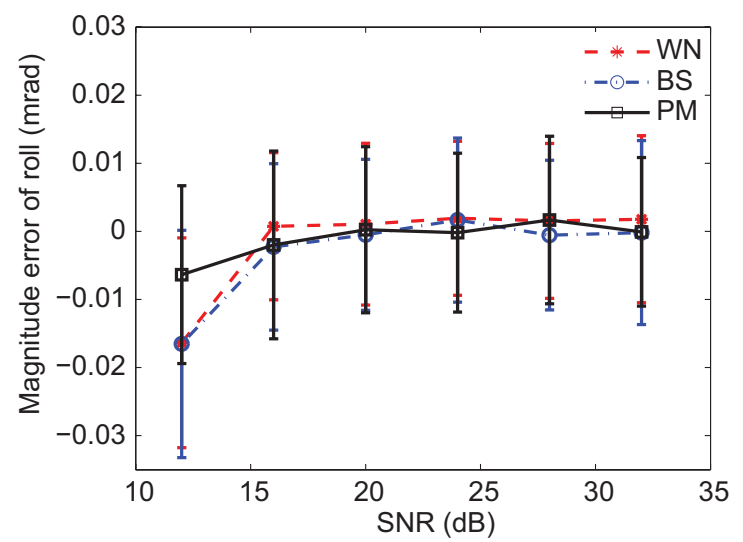

(b)

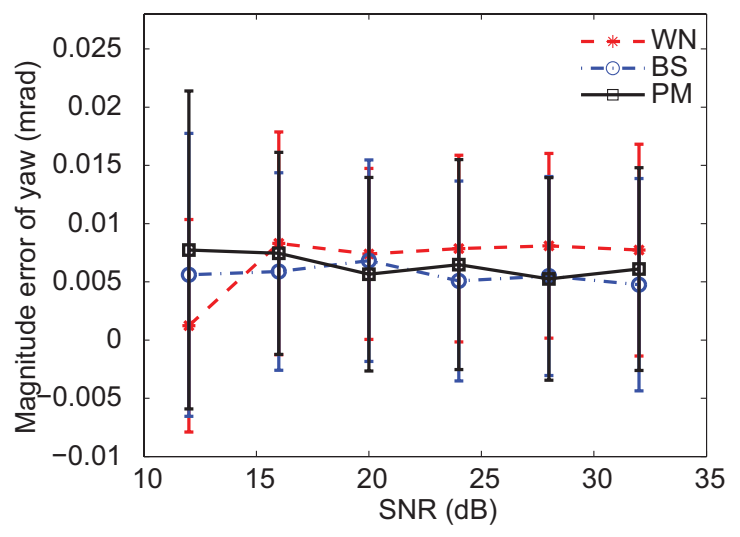

(c)

Fig. 6. Comparison of the estimation errors for magnitude $\sigma_{i}$ under different SNR values. (a) Pitch. (b) Roll. (c) Yaw. WN denotes white noise wave excitation force, and PM and BS denote colored noise wave excitation forces with PM and BS spectra, respectively.

\section{Discussion}

Our primary aim is to derive an online estimation method for identifying the dynamic flexure model parameters required by the KF-based TA algorithm. From the estimation results presented in Table $\mathrm{V}$ and Figs. 6-8, we observe that the estimates of the dynamic flexure parameters are consistently accurate over a wide range of SNR values as well as over different ship motion conditions. Therefore, our simulation results demonstrate that the proposed iterative TK estimation

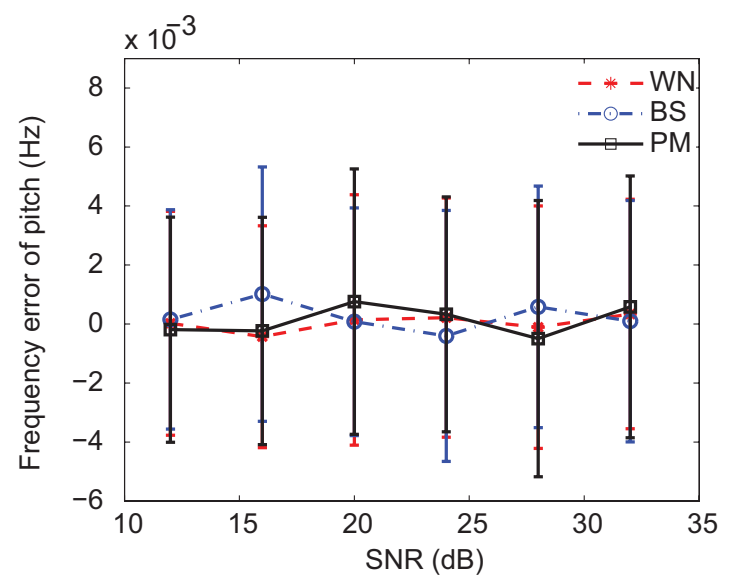

(a)

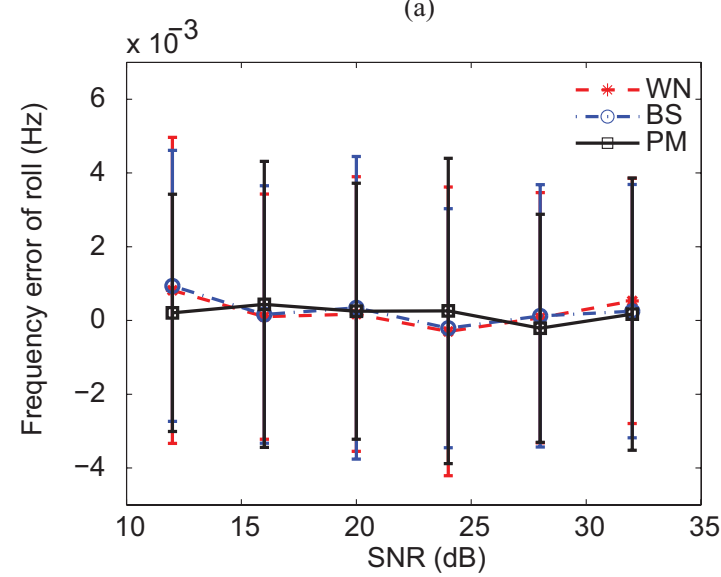

(b)

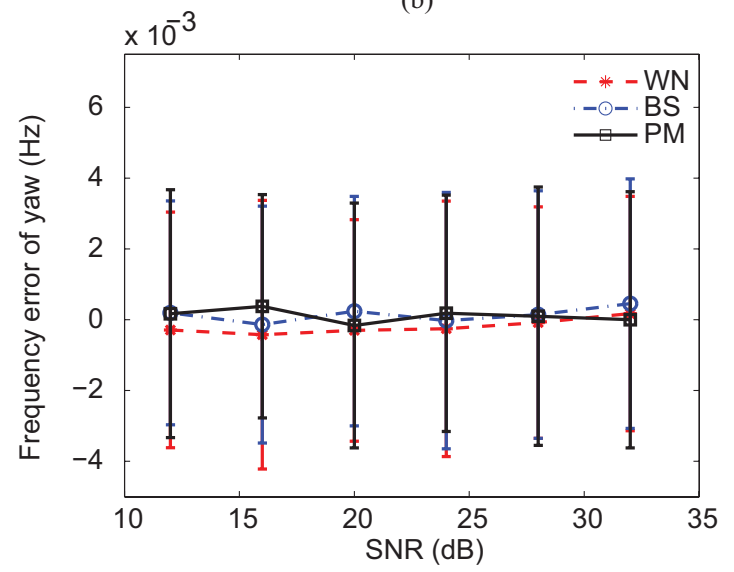

(c)

Fig. 7. Comparison of the estimation errors for frequency $\beta_{i} / 2 \pi$ under different SNR values. (a) Pitch. (b) Roll. (c) Yaw. WN denotes white noise wave excitation force, and PM and BS denote colored noise wave excitation forces with PM and BS spectra, respectively.

procedure is capable of obtaining robust and accurate estimates of the unknown dynamic flexure parameters, and we have achieved our primary objective.

The results of Table VI and Fig. 9 confirm that the KF-based TA achieves reasonably accurate alignment. However, we note that the alignment errors obtained under the ship motion conditions generated by the two colored noise wave excitation forces with the PM and BS spectra, respectively, are considerably larger than those obtained under the 


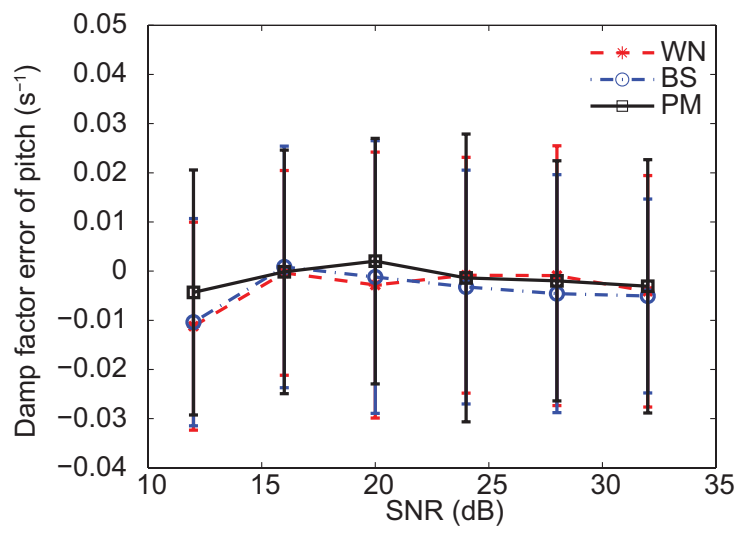

(a)

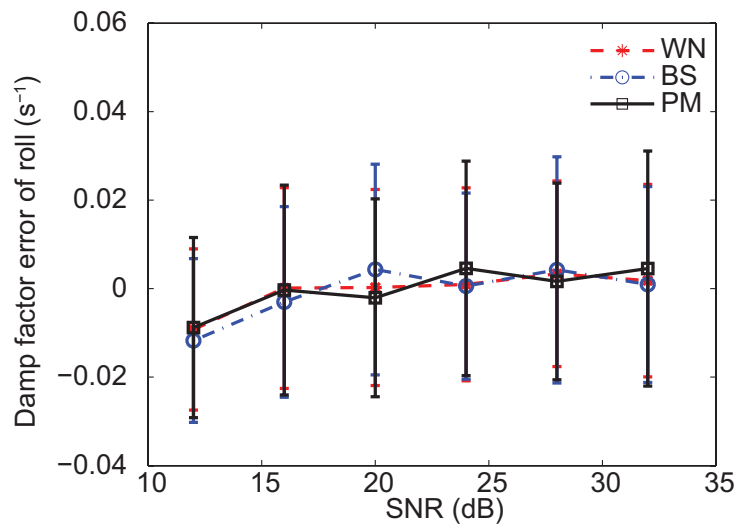

(b)

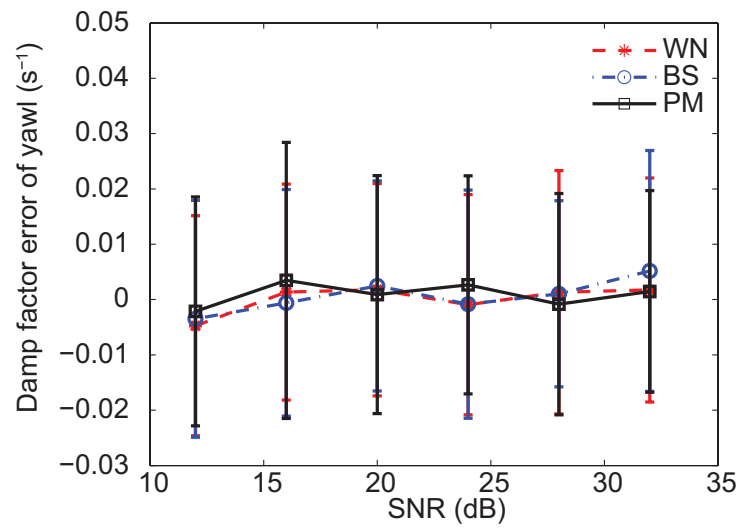

(c)

Fig. 8. Comparison of the estimation errors for the damping factor $\alpha$ under different SNR values. (a) Pitch. (b) Roll. (c) Yaw. WN denotes white noise wave excitation force, and PM and BS denote colored noise wave excitation forces with PM and BS spectra, respectively.

WN wave excitation force. This happens despite the fact that the dynamic flexure parameter estimates have the same high accuracy under these three ship motion conditions. This observation actually reflects a fundamental issue in angular velocity- or attitude-based TA, and warrants a detailed discussion.

The formulation of the existing standard angular rate matching method is established under the assumption that the ship's angular motion and the dynamic flexure are two uncorrelated processes [2], [4], [9], [10], [23]. When the ship's angular

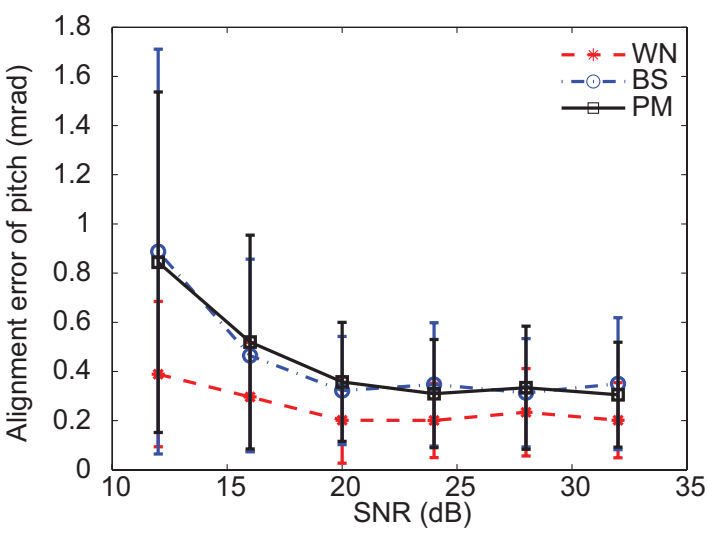

(a)

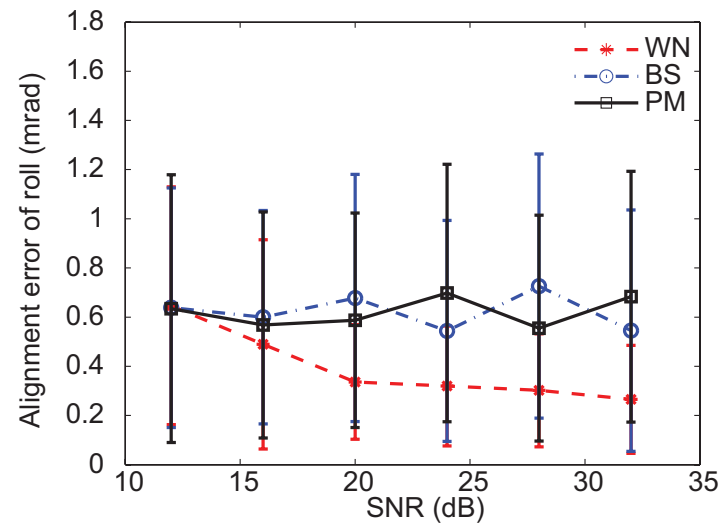

(b)

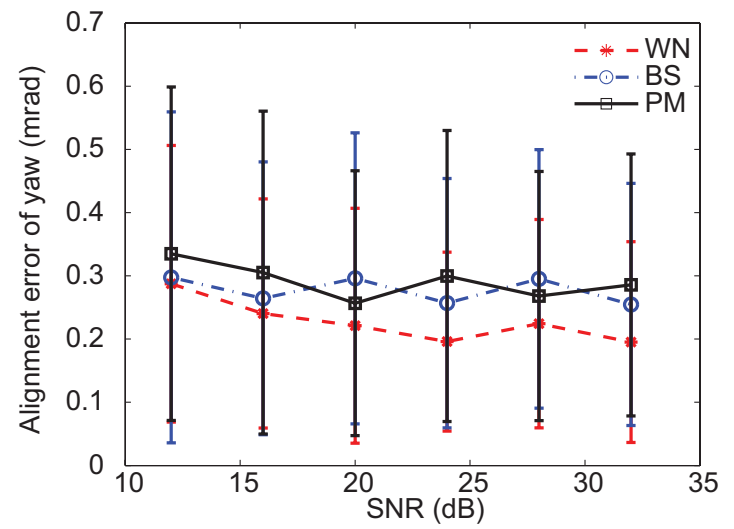

(c)

Fig. 9. Comparison of the KF-based TA alignment errors under different SNR values. (a) Pitch. (b) Roll. (c) Yaw. WN denotes white noise wave excitation force, and PM and BS denote colored noise wave excitation forces with PM and BS spectra, respectively.

motion and the dynamic flexure signal are all simulated using $\mathrm{WN}$ as driving or excitation forces, this assumption is met, and we observe the best alignment accuracy obtained under the simulation condition of the WN wave excitation force. When the wave excitation force used to simulate the ship's angular motion is colored, as in the cases of PM and BS spectra, the induced angular motion and the dynamic flexure becomes correlated, and we observe an increase in the alignment error, compared to the case where the ship's angular motion and the dynamic flexure are uncorrelated. 


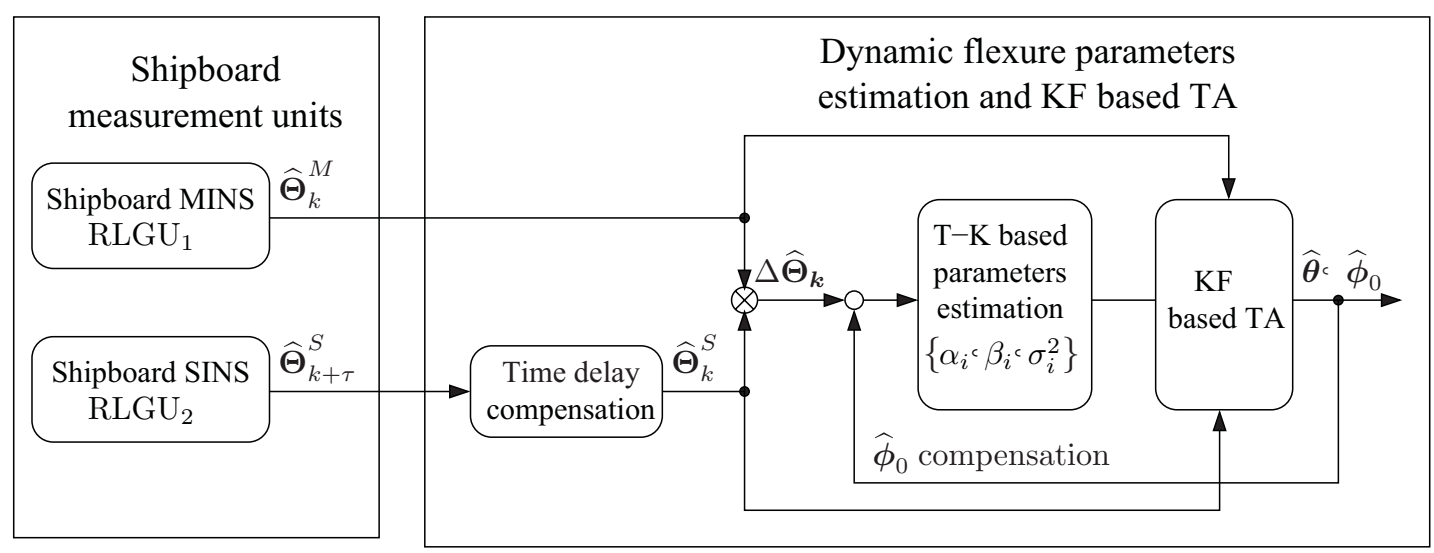

Fig. 10. Illustration of shipboard implementation of the proposed estimation algorithm for dynamic flexure parameters.

TABLE VI

Performance of The KF-BAsed TA With the Three Identified Dynamic Flexure Models Obtained Under the Noise-Free MEASUREMENT CONDITION OF $\sigma_{\varsigma_{i}}^{2}=0$ OR $\mathrm{SNR}_{i} \rightarrow \infty$

\begin{tabular}{l|c|c|c}
\hline \hline & $\begin{array}{c}\text { Mean (standard } \\
\text { deviation) of true } \\
\text { misalignment } \\
\text { angle (mrad) }\end{array}$ & $\begin{array}{c}\text { Mean (standard } \\
\text { deviation) of KF } \\
\text { estimated } \\
\text { misalignment } \\
\text { angle (mrad) }\end{array}$ & $\begin{array}{c}\text { Mean (standard } \\
\text { deviation) of KF } \\
\text { based alignment } \\
\text { error (mrad) }\end{array}$ \\
\hline \hline WN wave excitation force \\
\hline Pitch & $3.5102(0.4430)$ & $3.5180(0.4606)$ & $0.2069(0.1572)$ \\
Roll & $3.3917(0.7023)$ & $3.3667(0.7685)$ & $0.2674(0.1858)$ \\
Yaw & $3.4972(0.4829)$ & $3.4819(0.5496)$ & $0.1838(0.1128)$ \\
\hline \hline Colored noise wave excitation force with PM spectrum \\
\hline Pitch & $3.4312(0.3724)$ & $3.4343(0.5761)$ & $0.3722(0.2740)$ \\
Roll & $3.5539(0.7125)$ & $3.5832(1.0941)$ & $0.6756(0.4671)$ \\
Yaw & $3.5816(0.4754)$ & $3.6458(0.5395)$ & $0.2905(0.2015)$ \\
\hline \hline Colored noise wave excitation force with BS spectrum \\
\hline Pitch & $3.5353(0.3551)$ & $3.4921(0.5058)$ & $0.3433(0.2464)$ \\
Roll & $3.5386(0.6837)$ & $3.6019(0.8258)$ & $0.5423(0.4333)$ \\
Yaw & $3.4164(0.4936)$ & $3.4297(0.6478)$ & $0.2671(0.2296)$ \\
\hline \hline
\end{tabular}

It is worth emphasizing that the assumption of uncorrelated ship angular motion and dynamic flexure is in fact not very realistic. In real sea environments, the ship's angular motion and the dynamic flexure are the responses of the ship's structure to the wave loads and, therefore, they are likely to be correlated, in general. Thus, a coupling error is introduced by the projection of the additional dynamic flexure velocity on the ship angular velocity [2], [3]. This coupling influence will significantly affect the TA accuracy. It is therefore critical to study how to remove or reduce this coupling error in order to improve TA accuracy. This will be the focus of our future research.

We now briefly discuss the implementation of our proposed estimation method for the dynamic flexure parameters in real applications. Shipboard implementation of our parameter estimation algorithm is relatively straightforward, provided that the ship's MINS and SINS are all equipped with ring-laser gyro units, and are capable of taking the discretetime measurements of the angular increments $\widehat{\boldsymbol{\Theta}}^{M}$ and $\widehat{\boldsymbol{\Theta}}^{S}$ that are induced by the ship angular motion. These two measured signals are processed to achieve time synchronization, and they are then used by the iterative TK estimation procedure, instead of the simulated signals as shown in Fig. 2. Basically, the dynamic flexure parameters can be estimated directly using the measured gyro samples based on our proposed approach, as is illustrated in Fig. 10.

\section{CONCLUSION}

An online ship dynamic flexure parameter estimation method was proposed for the application to KF-based shipboard TA based on the MINS and SINS gyro outputs. The relationship between the correlation function of the angular increment difference and that of the second-order GaussMarkov process representing the dynamic flexure model was presented, and the TK method was applied to identify the unknown dynamic flexure model parameters. Our extensive simulation results demonstrated that the proposed online parameter estimation method can accurately estimate the unknown dynamic flexure model parameters, and consequently enable the KF to achieve high-accuracy alignment using the estimated dynamic flexure model, under the noise-polluted shipboard environment. Our results also showed that the parameter estimation algorithm is robust to the measurement noise and the ship motion conditions. As the proposed method does not require a priori knowledge of the dynamic flexure characteristics, it provides a rapid means for the KF to perform accurate shipboard TA.

\section{REFERENCES}

[1] C. B. Lawrence, "Master reference system for rapid at sea alignment of aircraft inertial navigation systems," in Proc. AIAA/JACC Conf. Guidance Control, Seattle, WA, Aug. 1966, pp. 593-606.

[2] B. H. Browne and D. H. Lackowski, "Estimation of dynamic alignment errors in shipboard firecontrol systems," in Proc. IEEE Conf. Decision Control, Clearwater, FL, Dec. 1976, pp. 48-57.

[3] A. V. Mochalov, "A system for measuring deformations of largesized objects," in Optical Gyros and their Application, D. Loukianov, R. Rodloff, H. Sorg, and B. Stieler, Eds. Moncton, NB, Canada: Canada Commun. Group, 1999, pp. 1-9.

[4] A. M. Schnider, "Kalman filter formulations for transfer alignment of strapdown inertial units," Navigation, vol. 30, no. 1, pp. 72-89, 1983. 
[5] D. L. Day and J. Arrud, "Impact of structural flexure on precision tracking," Naval Eng. J., vol. 111, no. 3, pp. 133-138, May 1999.

[6] M. G. Petovello, K. O'Keefe, G. Lachapelle, and M. E. Cannon, "Measuring aircraft carrier flexure in support of autonomous aircraft landings," IEEE Trans. Aerosp. Electron. Syst., vol. 45, no. 2, pp. 523535, Apr. 2009.

[7] C. Bacchus, I. Barford, D. Bedford, J. Chung, P. Dailey, S. Hill, J. Chung, R. Hazle, and M. Michocka, "Digital array radar for ballistic missile defense and counter-stealth systems analysis and parameter tradeoff study," Dept. Syst. Eng., Naval Postgraduate School, Monterey, CA, Tech. Rep. NPS-SE-06-001, Sep. 2009.

[8] A. V. Mochalov and A. V. Kazantasev, "Use of the ring laser units for measurement of the moving object deformation," in Proc. 2nd Int. Conf. Lasers Meas. Inf. Transfer, St. Petersburg, Russia, Jun. 2001, pp. 85-92.

[9] F. Sun, C. J. Guo, W. Gao, and B. Li, "A new inertial measurement method of ship dynamic deformation," in Proc. Int. Conf. Mechatron. Autom., Harbin, China, Aug. 2007, pp. 3407-3412.

[10] L. Joon and Y.-C. Lim, "Transfer alignment considering measurement time delay and ship body flexure," J. Mech. Sci. Technol., vol. 23, no. 1, pp. 195-203, 2009.

[11] D. Gebre-Egziabher and Y. Shao, "Model for JPALS/SRGPS flexure and attitude error allocation," IEEE Trans. Aerosp. Electron. Syst., vol. 46, no. 2, pp. 483-495, Apr. 2010.

[12] P. D. Groves, "Optimising the transfer alignment of weapon INS," $J$. Navigat., vol. 56, no. 2, pp. 323-335, May 2003.

[13] I. Watanabe and C. G. Soares, "Comparative study on the time-domain analysis of non-linear ship motions and loads," Marine Struct., vol. 12, no. 3, pp. 153-170, Apr. 1999.

[14] J. E. Kain and J. R. Cloutier, "Rapid transfer alignment for tactical weapon applications," in Proc. AIAA Conf. Guidance, Navigat. Control, Boston, MA, Aug. 1989, pp. 1290-1300.

[15] K. Spalding, "An efficient rapid transfer alignment filter," in Proc. AIAA Conf. Guidance, Navigat. Control, Head Island, LA, Aug. 1992, pp. 1276-1286.

[16] P. G. Shoals and D. E. Brunner, "Dynamic ship flexure measurement program," Naval Ship Weapon Syst. Eng. Station, Port Hueneme, CA, Tech. Rep. A047040, Aug. 1973.

[17] R. Kumaresan and D. Tufts, "Estimating the parameters of exponentially damped sinusoids and pole-zero modeling in noise," IEEE Trans. Acoust., Speech Signal Process., vol. 30, no. 6, pp. 833-840, Dec. 1982.

[18] N. Kannana and D. Kundu, "Estimating parameters in the damped exponential model," Signal Process., vol. 81, no. 11, pp. 2343-2351, Nov. 2001.

[19] T. Yu, "Improving the accuracy of parameter estimation for real exponentially damped sinusoids in noise," Proc. Inst. Elect. Eng. F, Radar Signal Process., vol. 137, no. 3, pp. 192-196, Jun. 1990.

[20] Y. Kim, B. W. Nam, D. W. Kim, and Y. S. Kim, "Study on coupling effects of ship motion and sloshing," Ocean Eng., vol. 34, no. 16, pp. 2176-2187, Nov. 2007.

[21] J. H. G. M. Alves, M. L. Banner, and I. R. Young, "Revisiting the Pierson-Moskowitz asymptotic limits for fully developed wind waves," J. Phys. Oceanogr., vol. 33, no. 7, pp. 1301-1323, Jul. 2003.

[22] N. Spidsöe and R. Sigbjörnsson, "On the reliability of standard wave spectra in structural response analysis," Eng. Struct., vol. 2, no. 2, pp. 123-135, Apr. 1980.

[23] S. Majeed and J. Fang, "Comparison of INS based angular rate matching methods for measuring dynamic deformation," in Proc. 9th Int. Conf. Electron. Meas. Instrum., Beijing, China, Aug. 2009, pp. 332-336.

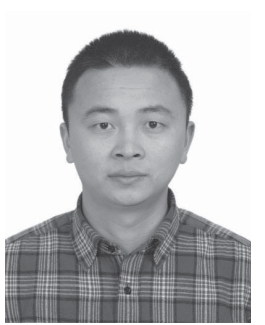

Wei Wu received the B.Sc. degree in applied physics from Northwestern Polytechnic University, Xi'an, China, in 2006, and the Master's degree in optical engineering from the National University of Defense Technology, Changsha, China, in 2008, where he is currently pursuing the Ph.D. degree with the School of Opto-Electronic Science and Technology.

$\mathrm{He}$ has been a Visiting Ph.D. Student with the Electronics and Computer Science Department, University of Southampton, Southampton, U.K., since October 2011. His current research interests include opto-electronic instrument measurement and control, system modeling, and identification.

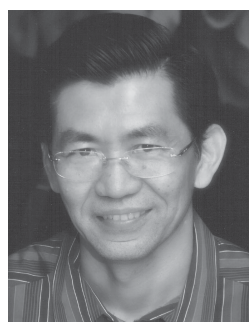

Sheng Chen (M'90-SM'97-F'08) received the B.Eng. degree from the East China Petroleum Institute, Dongying, China, in 1982, and the Ph.D. degree from City University, London, U.K., in 1986, both in control engineering, and the D.Sc. degree from the University of Southampton, Southampton, U.K., in 2005 .

He held research and academic appointments with the University of Sheffield, Sheffield, U.K., the University of Edinburgh, Edinburgh, U.K., and the University of Portsmouth, Portsmouth, U.K., from 1986 to 1999. Since 1999, he has been with the Electronics and Computer Science Department, University of Southampton, where he is currently a Professor of intelligent systems and signal processing. He is a Distinguished Adjunct Professor with King Abdulaziz University, Jeddah, Saudi Arabia. $\mathrm{He}$ is listed as one of the most highly cited researchers in engineering in the database of the world's most highly cited researchers compiled by the Institute for Scientific Information. He has authored or coauthored 450 research papers. His current research interests include adaptive signal processing, wireless communications, modeling and identification of nonlinear systems, neural network and machine learning, intelligent control systems design, evolutionary computation methods, and optimization.

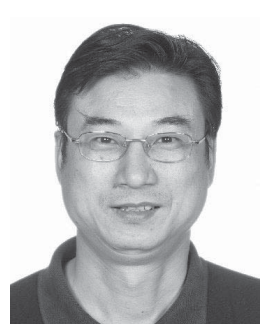

Shiqiao Qin received the B.Sc. degree from Wuhan University, Wuhan, China, in 1983, and the Master's and Ph.D. degrees in optical engineering from the National University of Defense Technology, Changsha, China, in 1986 and 1993, respectively.

$\mathrm{He}$ is currently a Professor with the School of Science, National University of Defense Technology. His current research interests include nano-optics, optical engineering, and opto-electronic instrument measurement and control. 